\title{
Presynaptic dysfunction in CASK-related neurodevelopmental disorders
}

\author{
Martin Becker $\mathbb{1}^{1,2}$, Francesca Mastropasqua ${ }^{1,2}$, Jan Philipp Reising ${ }^{2,3}$, Simon Maier ${ }^{4}$, Mai-Lan Ho ${ }^{5}$, lelyzaveta Rabkina ${ }^{1,2}$, \\ Danyang Li ${ }^{1,2}$, Janina Neufeld (10), Lea Ballenberger ${ }^{1,2,6}$, Lynnea Myers ${ }^{1,7}$, Viveka Moritz ${ }^{1,2}$, Malin Kele \\ Josephine Wincent ${ }^{9,10}$, Charlotte Willfors ${ }^{1,9}$, Rouslan Sitnikov ${ }^{11}$, Eric Herlenius $\mathbb{B}^{2,3}$, Britt-Marie Anderlid ${ }^{9,10}$, Anna Falk (10) ${ }^{8}$, \\ Sven Bölte $\mathbb{B D}^{1,12,13}$ and Kristiina Tammimies (B) $\mathbb{B}^{1,2}$
}

\begin{abstract}
CASK-related disorders are genetically defined neurodevelopmental syndromes. There is limited information about the effects of CASK mutations in human neurons. Therefore, we sought to delineate CASK-mutation consequences and neuronal effects using induced pluripotent stem cell-derived neurons from two mutation carriers. One male case with autism spectrum disorder carried a novel splice-site mutation and a female case with intellectual disability carried an intragenic tandem duplication. We show reduction of CASK protein in maturing neurons from the mutation carriers, which leads to significant downregulation of genes involved in presynaptic development and of CASK protein interactors. Furthermore, CASK-deficient neurons showed decreased inhibitory presynapse size as indicated by VGAT staining, which may alter the excitatory-inhibitory (E/l) balance in developing neural circuitries. Using in vivo magnetic resonance spectroscopy quantification of GABA in the male mutation carrier, we further highlight the possibility to validate in vitro cellular data in the brain. Our data show that future pharmacological and clinical studies on targeting presynapses and E/I imbalance could lead to specific treatments for CASK-related disorders.
\end{abstract}

\section{Introduction}

The identification of genetic variants underlying neurodevelopmental disorders (NDDs), such as intellectual disability (ID), autism spectrum disorder (ASD), and attention-deficit hyperactivity disorder (ADHD), as well as epilepsies, has increased at a rapid pace in recent years with a high level of pleiotropy across the conditions ${ }^{1-3}$. Findings indicate shared molecular mechanisms underlying the diverse clinical phenotypes. However, for many of the identified risk variants and genes, the molecular and neuronal outcomes are not well understood. One pleiotropic gene is the calcium/calmodulin-dependent serine protein kinase (CASK), located on chromosome Xp11.4, in

\footnotetext{
Correspondence: Kristiina Tammimies (kristiina.tammimies@ki.se)

${ }^{1}$ Center of Neurodevelopmental Disorders (KIND), Division of Neuropsychiatry, Department of Women's and Children's Health, Karolinska Institutet, and Center for Psychiatry Research, Region Stockholm, Sweden

${ }^{2}$ Astrid Lindgren Children's Hospital, Karolinska University Hospital, Region Stockholm, Sweden

Full list of author information is available at the end of the article
}

which pathogenic variants underlie a range of NDDs. Genetic variants in CASK were first described in cases with microcephaly with pontine and cerebellar hypoplasia (MICPCH), followed by the identification in cases with Xlinked ID (XL-ID), developmental delay (DD), and $\mathrm{ASD}^{1,4-6}$. The majority of cases reported with CASKrelated disorders are females with $\mathrm{MICPCH}$, caused by heterozygous loss-of-function (LoF) variants ${ }^{5,7}$. Skewed $\mathrm{X}$-chromosome inactivation $(\mathrm{XCI})$ has shown protective effects against more severe phenotypes ${ }^{5,8}$. Missense variants, found both in females and males, cause microcephaly, XL-ID, DD, or $\mathrm{ASD}^{1,4,6,8-10}$. The molecular pathways and cellular phenotypes associated with genetic variants causing CASK-related disorders are mostly unknown, especially in human neurons.

CASK is ubiquitously expressed with high expression in the developing human brain ${ }^{11}$. The protein structure consists of scaffolding domains L27, PDZ, and SH3, as well as a $\mathrm{Ca}^{2+} /$ calmodulin-dependent protein kinase and 
guanylate kinase domain ${ }^{12}$. In neurons, CASK is involved in pre- and postsynaptic signaling. At the presynapse, CASK regulates the synaptic vesicle exocytosis and neuronal cell adhesion through a tripartite complex with VELI1 (LIN7A) and MINT1 (APBA1) and direct interaction with NRXN1 $(N R X N 1)^{13,14}$. The tripartite protein complex VELI1-MINT1-CASK is unaffected by XL-ID and $\mathrm{MICPCH}$ missense mutations ${ }^{9}$. Instead, the CASK-NRXN1 interaction is disrupted, suggesting that the absence of this interaction is associated with the $\mathrm{MICPCH}$ phenotype. In the postsynaptic density, CASK contributes to the regulation of ionotropic receptor trafficking $^{15}$. Electrophysiological assessment of CASKnegative neurons from mice showed a shift in excitatory-inhibitory (E/I) balance with increased spontaneous miniature excitatory postsynaptic currents (mEPSC) and decreased miniature inhibitory postsynaptic currents (mIPSC) ${ }^{16,17}$. Interestingly, Nrxn1 KO mouse neurons and human neuronal models with NRXN1 LoF mutations have elevated CASK levels and decreased mEPSCs $^{18}$. In addition to the role at the synapse, CASK has been shown to act as a co-regulator of transcription through TBR1 (TBR1), CINAP (TSPYL2), and BCL11A $(B C L 11 A)^{12,19,20}$, all of which have been implicated in $\mathrm{NDDs}^{21-23}$.

Here, we elucidate the consequences of CASK pathogenic variants using human induced pluripotent stem cell (iPSC)-derived neuronal models from two mutation carriers, one diagnosed with ASD and one with $\mathrm{MICPCH}$. Our transcriptional, morphological, and functional analyses reveal that reduced levels of CASK are sufficient to induce aberrant presynaptic development, and have wide effects on its interaction network, as well as on multiple developmental pathways. In addition, for the first time, we explore the possibility to validate neuronal findings from iPSC models using in vivo brain data. In conclusion, our data suggest that CASK-related disorders are synaptopathies that affect the $E / I$ balance in maturing neural circuits.

\section{Materials and methods}

\section{Identification of mutation carriers}

The mutation carriers were recruited to the study from The Roots of Autism and ADHD study in Sweden (RATSS) and Clinical Genetics at the Karolinska University Hospital. Comprehensive phenotyping of the ASD case and his cotwin has been described elsewhere ${ }^{24,25}$. Phenotyping, genotyping, and clinical assessment of the girl with $\mathrm{MICPCH}$ was described earlier ${ }^{26}$. Written informed consent was obtained from affected individuals and their parents prior to the study. The study was approved by the regional and national ethical boards in Sweden, and it has been conducted in accordance with the Declaration of Helsinki for medical research involving human subjects, including research on identifiable human material and data.

\section{Whole-exome sequencing and data processing}

For the identification of the genetic alterations, we performed microarray analysis (for both cases) and wholeexome sequencing (for the twin pair and their parents). For de novo variant detection, the parents were included in the genetic analyses. DNA was extracted from whole blood and saliva samples using standard methods. The detection of CNVs has been described earlier ${ }^{26,27}$. Identification of clinically relevant variants from WES is detailed in the Supplementary material and methods. Categorization was made based on the criteria from the American College of Medical Genetics and Genomics $(\mathrm{ACMG})^{28}$.

\section{Cell culture of iPS and NES cells}

Human iPSC cells were derived for the CASK-mutation carriers diagnosed with $\mathrm{MICPCH}$ and ASD, using a previously described procedure ${ }^{29}$. A summary of the protocol and quality control of the iPS cells is described in the Supplementary materials and methods. Dual-SMAD inhibition was applied to derive NES cells from human iPS cells as described previously ${ }^{30,31}$.

NES cells were seeded on plastic surface, precoated with $20 \mu \mathrm{g} / \mathrm{ml}$ poly-ornithine (Sigma Aldrich) and $1 \mu \mathrm{g} / \mathrm{ml}$ laminin (Sigma Aldrich) in DMEM/F12 + glutamax medium (Gibco) supplemented with 0.5× B-27 (Gibco), $1 \times \mathrm{N}-2$ (Gibco), and $10 \mathrm{U} / \mathrm{ml}$ penicillin/streptomycin (Gibco). Cells were maintained in a $5 \% \mathrm{CO}_{2}$ atmosphere at $37^{\circ} \mathrm{C}$. Two-third of the medium was changed every second day of differentiation with $0.4 \mu \mathrm{g} / \mathrm{ml}$ laminin added. Neurons were differentiated for 8,16 , or 28 days. Cells harvested for time point day 0 were cultured for 2 days in NES cell medium.

\section{RNA extraction and RT-qPCR}

Cells were lysed in TRIzol reagent (Invitrogen), and RNA was isolated using the ReliaPrep RNA Cell Miniprep (Promega \#A1222). The RNA was reverse-transcribed using iScript cDNA Synthesis Kit (BioRad) and cDNA quantified with SsoAdvanced Universal SYBR Green Supermix (BioRad) following the manufacturer's protocols on a CFX96 thermal cycler (BioRad). CFX Manager software was used to record amplification curves and to determine $\mathrm{Ct}$ values. RT-qPCR reactions were performed in technical triplicates. We calculated the $\Delta \mathrm{Ct}$ to the GAPDH housekeeping gene and ${ }^{\Delta \Delta} \mathrm{Ct}$ to control cell lines. We used three biological replicates of cells seeded at different passages, if not stated otherwise in the figure legends. Statistical significance between cell lines was determined with ANOVA and post hoc Tukey HSD in R. 


\section{Protein quantification using capillary western blot}

Cells were dissociated in extraction buffer ( $50 \mathrm{mM}$ Tris$\mathrm{HCl}, 100 \mathrm{mM} \mathrm{NaCl}, 5 \mathrm{mM}$ EDTA, and $1 \mathrm{mM}$ EGTA) using plastic cell scraper and lysed with 6 short sonication bursts at 36\% amplitude (Vibra-Cell VCX-600, Sonics). About $250 \mu \mathrm{g} / \mathrm{ml}$ protein was loaded for Simple-Western WES (ProteinSimple) quantification with antibodies for CASK (1:500 Novus Biologicals NBP2-41181), beta-actin (1:100 Abcam ab8227), and GAPDH (1:5000 Sigma G9545). Compass Software For Simple Western (Version 4.0.0) was used to identify protein-specific peaks. In the chromatogram, the peak area was used for protein quantification. We normalized CASK protein for housekeeping protein. For time point days 0,8 , and 16, we obtained two and three biological replicates for time point 28 and siRNA-mediated knockdown of CASK. Biological replicates were seeded at different dates, using different passages. Statistical significance between cell lines was determined with ANOVA and post hoc Tukey HSD in R.

\section{Immunofluorescence}

Cells were cultured on glass coverslips for the indicated differentiation time and fixed for $20 \mathrm{~min}$ in $4 \%$ formaldehyde. Primary antibodies used are CASK-NBP241181, 1:500 (Novus bio), MAP2-M2320, 1:500 (Sigma Aldrich), VGLUT1-135304, 1:250 (Synaptic systems), Homer-1-160011, 1:250 (Synaptic systems), VGAT131003, 1:500 (Synaptic systems), Synapsin-1/2-106006, 1:500 (Synaptic systems), Gephyrin-147021, 1:250 (Synaptic systems), Nestin-MAB5326-KC, 1:1000 (MerckMillipore), and SOX2-AB5603, 1:1000 (Merck-Millipore). All images were taken with LSM 700 Zeiss Confocal Microscope (Zeiss Plan-Apochromat 63×/1.40na Oil DIC Objective M27), with $63 \times$ magnification at 1024- $\times 1024-$ pixel [pxl] resolution, resulting in an aspect ratio of $0.099233 \mu \mathrm{m}$ per pixel.

ImageJ Particle Analyzer was used to count CASK puncta and cell nuclei. Quantification of particle number and size was done in R. Four independent replicates, seeded at different dates and passages, were obtained for each cell line. Synaptic marker particle size and number were quantified with the ImageJ plugin Synapse Counter, using default settings ${ }^{32}$. For the Synapsin-1/2-Homer-1 and VGlut-Homer-1 co-staining, we obtained four independent replicates for each cell line. For the VGAT-Homer-1 and VGAT-Gephyrin co-staining, we obtained three independent replicates.

\section{siRNA-mediated gene knockdown}

NES cells were seeded for differentiation and the next day transfected with $0.5 \mu \mathrm{M}$ Accell SMARTpool siRNA targeting CASK mRNA (Dharmacon \#E-005311-00-0010) or $0.5 \mu \mathrm{M}$ Accell Green Nontargeting siRNA (Dharmacon \#D-001950-01-20) according to standard protocol. Three biological replicates were harvested for each protein and RNA analysis.

\section{Single-cell RNA sequencing}

Cells were dissociated from culture surface with $2 \mathrm{~min}$ of trypsin incubation and subsequent trypsin inhibition. After $3 \mathrm{~min}$ centrifugation at $300 \mathrm{rcf}$, the cells were resuspended in cold Dulbecco's phosphate-buffered saline, and single cells sorted by size into lysis buffer using BD FACS Aria III. Smart-Seq2 library preparation and sequencing were done with the Eukaryotic Single Cell Genomics (ESCG) facility in the SciLifeLab, Stockholm ${ }^{33}$. In total, 384 sorted wells were sequenced with a total of 209.6 M reads and an average sequence depth of 550,000 reads per cell. The individual counts per gene and cell were reported in a count matrix (GSE140572) and used for further analysis. The scImpute package was used to calculate dropout expression values from the count matrix $^{34}$. Cells with less than 50,000 read counts in less than 2000 genes were removed from analysis. Differential gene expression and cell clustering were done using the Seurat package ${ }^{35}$. To obtain mutant CASK reads, we created a custom genome with STAR that included the cDNA sequence of the CASK gene with the duplicated exons 4 and 5 and realigned all sequence reads. Exon 5-exon 4 spanning sequence reads were analyzed using vcftools.

\section{Bulk RNA sequencing}

We extracted RNA samples of five biological replicates per cell line that were seeded at different dates of different passages. Samples were delivered to NGI Sweden for library preparation and sequencing. We obtained on average 50.4 million reads per sample with a minimum of 96.9\% reads aligned to protein-coding regions. Sample read counts are supplied in GSE140572. Replicates of each cell line cluster together with the exception of one $\mathrm{MICPCH}$ replicate that was removed from analysis.

Differential gene expression was calculated from gene counts using DESeq2 (v1.24.0) in R. To determine a differential expression for individual cases, we used passage and cell lines as independent variables and compared ASD $_{\text {CASK_SS }}$ with male control and $\mathrm{MICPCH}_{\text {CASK_dup } 4 / 5}$ with female control. Top ranking genes for comparison between ASD $_{\text {CASK_Ss }}$ and $\mathrm{MICPCH}_{\mathrm{CASK} \text { dup } 4 / 5}$ were filtered at an adjusted $p$ value of $1 \mathrm{E}-5$ (BHA), with more than 20 reads and $\log 2$ fold change (LC) greater than 0.5 . The Venn diagram was visualized in $\mathrm{R}$ using the VennDiagramm package (v1.6.20), and overrepresentation analysis was performed using WebGestaltR (v0.4.2).

Pathway analysis was done according to previously described protocols ${ }^{36}$. In short, the ranked gene expression list was used in Gene set enrichment analysis (GSEA) (Version 3.0) ${ }^{37}$ and enriched categories were visualized in 
Cytoscape (v3.7.0) with Enrichment Map (v3.1.0) and AutoAnnotate ${ }^{38,39}$. In addition, GSEA was performed on CASK PPI (downloaded from PathwayCommons; PCViz: CASK on 08.Oct.2019), an in-house NDD gene list (Supplementary Material and Methods), and SFARI sublist.

\section{Deconvolution}

The deconvolution of bulk RNASeq data was done using the BSEQ-sc package ${ }^{40}$. BSEQ-sc uses cell-typespecific marker genes from single-cell RNA transcriptomes to predict cell-type proportions underlying bulk RNA transcriptomes. Deconvolution was done on increasing numbers of significant genes, and the predictions were stable when using 10 or 20 most significant marker genes. Statistical differences in the estimated cell proportions between patient and both control cell lines were done using ANOVA and post hoc Tukey HSD test.

\section{Calcium imaging}

Calcium imaging was performed according to protocols described earlier ${ }^{41}$. Cells were loaded with Fluo-8 for $45 \mathrm{~min}$ at $37^{\circ} \mathrm{C}$ with $5 \% \mathrm{CO}_{2}$ and imaged for $5 \mathrm{~min}$ at $37^{\circ} \mathrm{C}$ at normal atmosphere. Mean gray values of ROIs were recorded over the 5-min preprocessed time series to obtain traces of neuronal activity. Traces were resampled to $2 \mathrm{~Hz}$ and smoothed using the python scipy.signal package. Peaks were detected using the PeakCaller Matlab software $^{42}$. Biological replicates at 4 weeks: female control $(n=2)$, male control $(n=2), \operatorname{ASD}_{\mathrm{CASK} \_S S}(n=1)$, and $\mathrm{MICPCH}_{\mathrm{CASK} \_d u p 4 / 5}(n=2)$. Five weeks: female control $(n=2)$, male control $(n=2), \operatorname{ASD}_{\text {CASK_SS }}(n=5)$, and $\mathrm{MICPCH}_{\text {CASK_dup } 4 / 5}(n=2)$. Active cells per cell line were pooled across replicates, and statistical differences at 4 and 5 weeks were calculated using pairwise Wilcoxon Rank Sum test followed with Bonferroni correction.

\section{[1H]MRS}

Proton magnetic resonance spectroscopy ([1H]MRS) data were available from the EU-AIMS Longitudinal European Autism Project (LEAP) autism twin cohort, including $\mathrm{ASD}_{\text {CASK_SS }}{ }^{43}$. MR data were acquired on MR750, 3 Tesla scanner (GE Healthcare, Milwaukie, USA), with 8-channel receiver array coil (in-VIVO Inc.). MRS data were acquired with MEGA-PRESS pulse sequence, $\operatorname{te} / \mathrm{tr}=68 / 2000 \mathrm{~ms}$, number of averages 192 , phase cycle 8 steps, and bandwidth $5 \mathrm{kHz}$ for three voxels with volumes $19.3 \mathrm{~mL}$ (Medial PFC), $19.5 \mathrm{~mL}$ (PutamenGPS), and $13.6 \mathrm{~mL}$ (DLPFC). GABA concentration of all three voxels was quantified from the MEGA-PRESS difference spectra using the "Gannet" GABA-MRS analysis toolkit, version $3.0^{44}$. Metabolite values were scaled to water and expressed in institutional units (IU). Waterscaled GABA concentrations represent GABA as well as related macromolecules, and are therefore referred to as GABA + concentrations. GABA concentrations were adjusted for the average proportion of partial GM and white matter (WM) volume in each voxel across all subjects. For tissue correction, the GABA concentration ratio $\alpha$ between GM and WM was set to $0.5^{45}$. All spectra were visually evaluated for the quality of GABA fit. For the final analysis, 45 spectra from the Putamen (20 cotwins to individuals with NDDs), 33 from the MFC (11 cotwins to individuals with NDDs), and 44 from the DLPFC (19 cotwins to individuals with NDDs) of the control subjects could be included.

\section{Results \\ CASK variants lead to mutant transcripts and reduced CASK $_{\mathrm{WT}}$ expression}

To analyze the converging molecular and cellular consequences of pathogenic variants affecting CASK across the associated NDD spectrum, we recruited two families with unique $C A S K$ genetic alterations, representing ASD and $\mathrm{MICPCH}$ phenotypes. First, we identified a male individual diagnosed with ASD carrying a CASK mutation from the Roots of Autism and ADHD Twin Study in Sweden (RATSS) ${ }^{24}$. He had a primary diagnosis of ASD and general intellectual abilities in the upper average range without cognitive impairments (total $\mathrm{IQ}=121$ ) and additional diagnoses of ADHD and tic disorder (Fig. 1a, Supplementary Table 1). His monozygotic cotwin was diagnosed with ASD prior to the RATSS study. However, the cotwin did not fulfill clinical consensus ASD diagnostic criteria at reassessment during the study, but still exhibited autistic symptoms (Supplementary Table 1). Anatomical magnetic resonance imaging (MRI) scans of both twins revealed mild cerebral atrophy and mild cerebellar hypoplasia within normal variation (Supplementary Fig. 1a, b). No clinically significant copy number variants were found in the twin pair. Rare variants from the exome sequencing were prioritized based on variant effect, inheritance mode, and genes involved in NDDs. We identified a novel maternally inherited splice-site variant in intron 14 of the CASK gene in both twins (chrX: 41,586,906 C>A (hg38); NM_001126055: c.1296+1 G>T, Fig. 1a, Supplementary Fig. 1c). The variant disrupts the consensus splice donor site at $+1 \mathrm{G}$ nucleotide (Supplementary Fig. 1d). CASK is intolerant to LoF mutations $(\mathrm{pLI}=1)$, and three putative LoF splice-site mutations reported in the general population are outside the main transcript or tissue-specific transcripts, as reported by the GTEx consortium ${ }^{46,47}$. In addition, both twins carry a de novo heterozygous missense mutation affecting DIAPH1 exon 15 (chr5: 141,574,906 C>A (hg38); NM_005219: c.1528 G>A: p.E510K, Supplementary Fig. 1e). Heterozygous truncating mutations of this gene are reported in cases of congenital deafness ${ }^{48}$, and homozygous LoF 


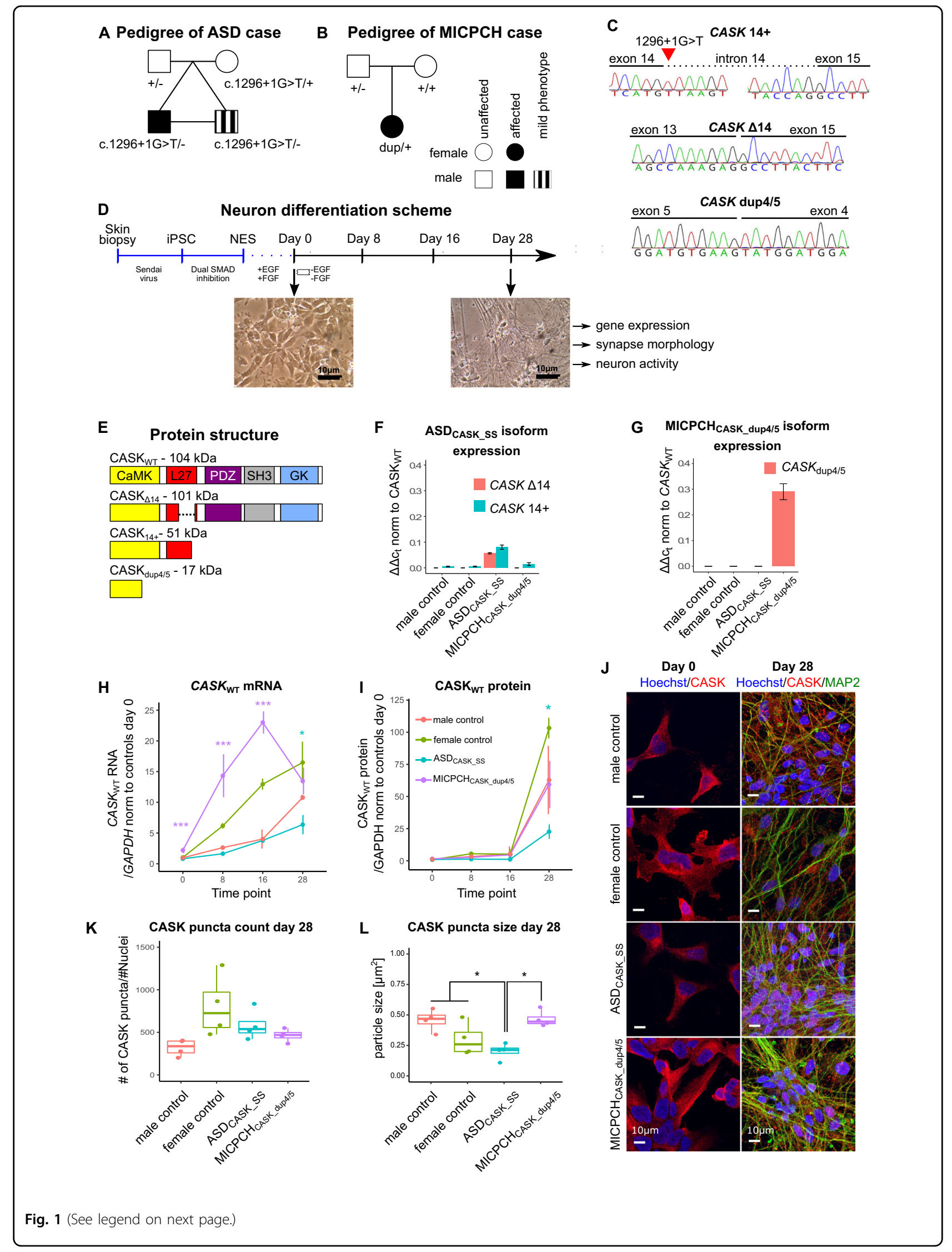


(see figure on previous page)

Fig. 1 CASK mutations affect wild-type expression in carriers. a Pedigree of a male diagnosed with ASD and his monozygotic cotwin who exhibits autistic traits, both carrying a splice-site mutation in the X-linked CASK gene (chrX: 41,586,906 C>A (hg38); NM_001126055: c.1296+1 G>T) inherited from a typically developed mother. $\mathbf{b}$ Pedigree of a female diagnosed with MICPCH, carrying a de novo duplication of $54.9 \mathrm{~kb}$ surrounding exons 4 and 5 (chrX:41710324-41765176 (hg38)) of the CASK gene. $\mathbf{c}$ Sanger chromatogram of CASK mRNA isoforms in CDNA of ASD(AASK_SS fibroblasts and MICPCH representative phase-contrast microscopy images of male control NES cells and day 28 neurons. e Protein domain structure of CASK $K_{W T}$ and in silico-

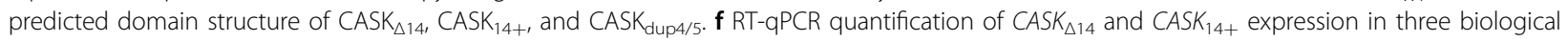
replicates of case and control NES cells. $\mathbf{g}$ RT-qPCR quantification of CASK dup $4 / 5$ in three biological replicates of case and control NES cells. $\mathbf{h}$ RT-qPCR quantification of CASK $K_{W T}$ in three biological replicates of differentiating neurons. i Protein quantification of CASK $K_{W T}$ protein in two biological replicates of differentiating neurons using capillary western blot quantification. j Representative confocal microscopy images of NES cells (day 0 ) and differentiated neurons (day 28) immunostained for CASK (red), MAP2 (green), and Hoechst (blue). Scale $=10 \mu m$. k, I Quantification of CASK puncta $\mathbf{k}$ number and I size from confocal images ( $n=4$ per cell line). Statistical differences between cases and controls were calculated using ANOVA with post hoc Tukey HSD. ${ }^{*} p<0.05,{ }^{* * *} p<0.001$. Asterisks in $\mathbf{a}$ and $\mathbf{b}$ are color-coded according to case cell lines.

mutations are linked to microcephaly ${ }^{49}$. None of the reported dominant mutations in DIAPH1 have been linked to NDDs. Of interest, slight hearing problems in one ear were reported for one of the twins (Supplementary Table 1).

We also recruited a female with a classical presentation of MICPCH syndrome, caused by a de novo duplication of $54.9 \mathrm{~kb}$ at chromosome Xp11.4, spanning two exons of $\operatorname{CASK}^{26}$ (Fig. 1b). Her degree of ID is in accordance with earlier-described individuals with MICPCH. She did not communicate verbally, had pontocerebellar hypoplasia, scoliosis, optic nerve hypoplasia, and infantile spasms and epilepsy. Wincent et al. provided detailed description of the individual (referred to as patient 7 in their study), including clinical symptoms, structural brain MRI, and genetic analysis.

To investigate the consequences of the identified splicesite and duplication variants to CASK mRNA, we obtained fibroblasts from the two mutation carriers hereafter referred to as $\mathrm{ASD}_{\mathrm{CASK} \_S s}$ and $\mathrm{MICPCH}_{\mathrm{CASK} \_d u p 4 / 5}$. The obtained fibroblasts were transformed into iPSCs. The iPSCs had pluripotent marker expression, normal karyotype, and matched donor genotypes (Supplementary Fig. 1f, g). Furthermore, the iPSCs were differentiated to long-term self-renewing neuroepithelial-like stem cells (further referred to as NES cells, Fig. 1d) ${ }^{31}$. NES cell lines derived from two sex-matched typically developed individuals were obtained for controls ${ }^{29,31}$. We detected two mutant CASK mRNA isoforms in the NES cells from ASD $_{\text {CASK_SS }}$ (Fig. 1c). One isoform lacks exon 14 $\left(\right.$ CASK $\left._{\Delta 14}\right)$, and one isoform retains 196 nucleotides of intron $14\left(\mathrm{CASK}_{14+}\right)$, resulting in a premature stop codon (Fig. 1e). Although ASD $_{\text {CASK_Ss }}$ is hemizygous, we detected wild-type CASK mRNA (CASK ${ }_{\mathrm{WT}}$ ) with intact joining of exons 14 and 15 constituting the majority of the expression. A minor proportion of CASK mRNA consisted of mutant $\mathrm{CASK}_{\Delta 14}$ and $\mathrm{CASK}_{14+}$ isoforms with approximately $6 \%$ and $8 \%$, respectively (Fig. 1 f). CASK $_{14+}$ isoform was detected at low levels in control and
MICPCH samples, presenting likely pre-mRNA. The presence of $\mathrm{CASK}_{\mathrm{WT}}$ in the cells of $\mathrm{ASD}_{\mathrm{CASK} \text { SS }}$ indicates rescue mechanisms for normal splicing possibly through mRNA editing. The $\mathrm{MICPCH}_{\mathrm{CASK}_{\text {dup } 4 / 5}} \mathrm{NES}$ cells expressed an mRNA isoform with a tandem duplication of exons 4 and 5 (Fig. 1c), which leads to a frame shift and early stop codon in the CASK coding sequence (Fig. 1e). This mutant isoform constitutes $\sim 29 \%$ of total CASK mRNA in $\mathrm{MICPCH}_{\mathrm{CASK} \_d u p 4 / 5}$ cells (Fig. $1 \mathrm{~g}$ ). In summary, we show that NES cells from both mutation carriers express $\mathrm{CASK}_{\mathrm{WT}}$ and detectable levels of mutant mRNA isoforms that may interfere with CASK function if translated to protein.

\section{Reduction of CASK expression in maturing neurons from mutation carriers}

Next, we differentiated NES cells to neurons and investigated the neurodevelopmental expression of CASK variants in vitro. To cover changes occurring during transition from neuroepithelial stem cells to maturing neurons, we collected RNA and protein from 8, 16, and 28 days of neuronal differentiation (Fig. 1d). None of the predicted mutant protein isoforms were detected from capillary western blotting using the N-terminal antibody (Supplementary Fig. 2a), suggesting that mutant mRNAs are removed by nonsense-mediated decay (NMD). CASK mRNA and protein expression increased with differentiation in all cell lines (Fig. 1h, i). However, at 28 days of differentiation, the $\mathrm{ASD}_{\mathrm{CASK}_{\text {SS }}}$ cells expressed significantly reduced levels of CASK mRNA and protein in comparison to both controls $(p=0.02$ and $p=0.01$, respectively, ANOVA post hoc Tukey). mRNA but not protein levels were increased in the $\mathrm{MICPCH}_{\text {CASK_dup4/5 }}$ from NES stage to day 16 and leveled with controls on day 28 (day $0 p<0.001$, day $8 p<0.001$, day $16 p=0.001$, and day $28 p=1$, ANOVA post hoc Tukey). The protein levels at day 28 are reduced in comparison with the sexmatched female control $(p=0.04$, ANOVA post hoc Tukey, Supplementary Fig. 2b). In addition to NMD, 

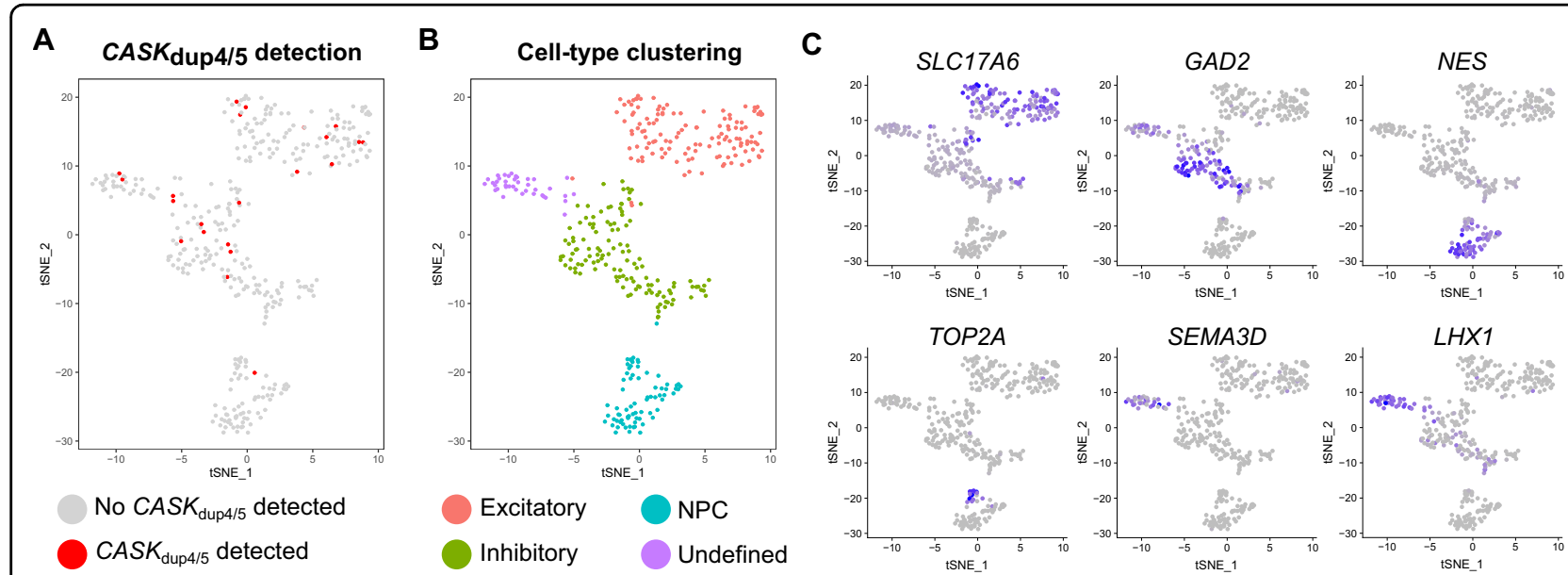

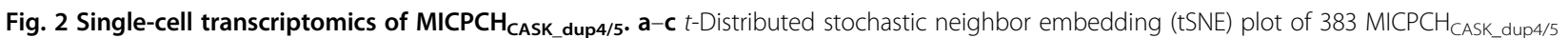
cell transcriptomes after 28 days of differentiation colored by a the detection of mutant CASK dup $4 / 5$ expression, $\mathbf{b}$ the identified cell-type clusters, and c expression of SLC17A6, GAD2, NES, TOP2A, SEMA3D, and LHX1.

biallelic expression from both $\mathrm{X}$ chromosomes may explain the discrepancy between increased CASK mRNA and normal protein levels in $\mathrm{MICPCH}_{\text {CASK_dup } 4 / 5}$.

We further investigated the cellular localization of CASK protein in the $\mathrm{ASD}_{\text {CASK_SS }}$ and $\mathrm{MICPCH}_{\text {CASK_dup4/5 }}$ cells using the N-terminal binding antibody. In NES cells, we observed small CASK puncta in the cytoplasm of all cell lines with no aberrant nuclear staining (Fig. 1j, day 0). Spatial localization of CASK protein as puncta in neurons has been described earlier ${ }^{50}$. With continuing differentiation, the CASK puncta remained in the cytoplasm, including the neurites (Fig. 1j, day 28) with comparable localization in all cell lines. In $\mathrm{MICPCH}_{\mathrm{CASK} \_d u p 4 / 5}$, CASK staining was detected in all cells demonstrating that the $C A S K_{\mathrm{WT}}$ allele is active in all cells. Quantification of CASK puncta showed similar numbers per nuclei $(p=0.99$ ASD $_{\text {CASK_Ss }}$ vs. controls, $p=0.83 \mathrm{MICPCH}_{\text {CASK_dup } 4 / 5}$ vs. controls, ANOVA post hoc Tukey, Fig. 1k) with puncta significantly smaller in differentiated ASD $_{\text {CASK_ss }}$ neurons compared to controls and $\mathrm{MICPCH}_{\mathrm{CASK} \_ \text {dup } 4 / 5}(p=0.049$ and $p=0.011$, respectively, ANOVA post hoc Tukey, Fig. 1l). We did not observe significant differences in MICPCH${ }_{\text {CASK_dup } 4 / 5}$ neurons compared to controls.

\section{X-chromosome activity in $\mathrm{MICPCH}_{\text {CASK_dup4/5 }}$ cells}

Random XCI was tested in blood from the $\mathrm{MICPCH}_{\mathrm{CASK} \_d u p 4 / 5}$ case in the clinical setting showing a relation between $24 \%$ and $76 \%$, consistent with random inactivation. We hypothesized that the discrepancy between CASK mRNA and protein expression during differentiation was due to biallelic expression from wild-type and mutant alleles. To investigate XCI and escape of CASK mRNA in our model, we performed SMART-Seq2 single-cell RNA sequencing on
$\mathrm{MICPCH}_{\mathrm{CASK} \_d u p 4 / 5}$ neurons differentiated for 28 days. We obtained quality reads for 383 cells with a total of $209.6 \mathrm{M}$ reads and an average sequence depth of 550,000 reads per cell. Approximately, $80 \%$ of uniquely aligned reads aligned to the genome, $40 \%$ to exons, and $30 \%$ to introns (Supplementary Fig. 3a, b). We identified the $\mathrm{CASK}_{\mathrm{dup} 4 / 5}$ specific exon 5-exon 4 junction in 22 cells (Fig. 2a, Supplementary Fig. 3c). We compared this detection rate to the detection of the adjacent exons 4 (62 cells) and 5 (51 cells), indicating that $35-43 \%$ of cells expressed $\mathrm{CASK}_{\mathrm{dup} 4 / 5}$. The frequency of CASK $_{\text {dup } 4 / 5}$-expressing cells is indicative of random $\mathrm{XCI}$ and comparable to detection levels of the mutant isoform in RT-qPCR (Fig. 1g). The presence of wildtype protein in all $\mathrm{MICPCH}_{\mathrm{CASK}_{\text {dup }} / 5}$ cells hints toward the escape of $C A S K_{\mathrm{WT}}$ from XCI. As cases and animal models of MICPCH do not show escape of $C A S K_{W T}$ in primary tissue samples, XCI escape is likely an artifact and the phenotype of our cell model milder than that of the patient.

Moreover, we identified cell types in the differentiated neuronal culture from the single-cell transcriptomes and mapped $C A S K_{\text {dup } 4 / 5}$ expression. The cultured cells clustered into four neuronal subtypes, which we identified as neural progenitor cells (NPC), excitatory and inhibitory neurons, as well as one cluster of undefined maturing neurons (Fig. 2b). Excitatory neurons express the SLC17A6 gene encoding for the vesicular glutamate transporter VGLUT2, and inhibitory neurons express the GAD2 gene encoding the GAD65 enzyme required for GABA production (Fig. 2c). NPCs express neuronal progenitor marker Nestin (NES) and cell-cycle gene TOP2A. Selective markers of the fourth cluster included genes involved in axon guidance SEMA3D and LHX1. 


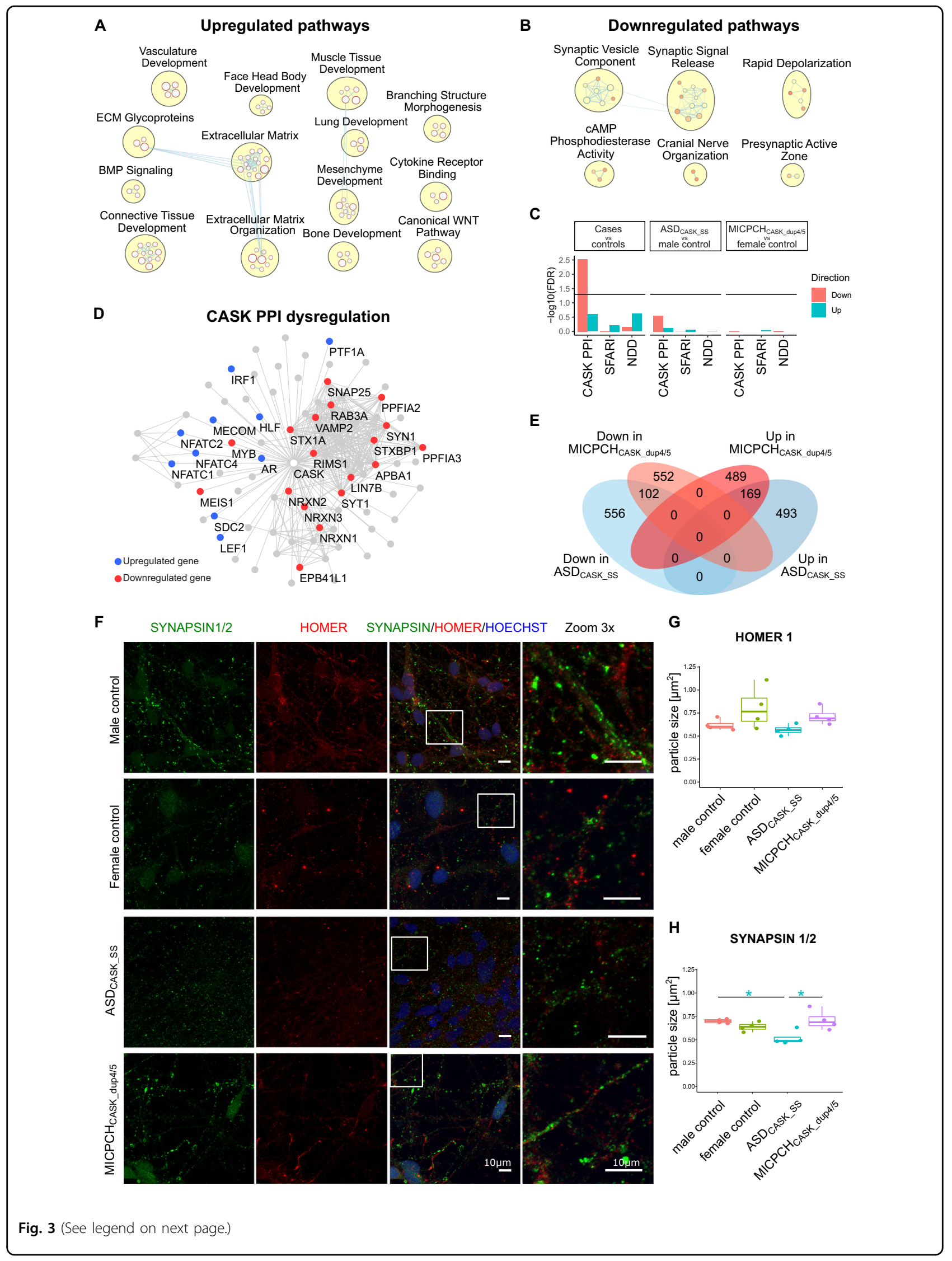


(see figure on previous page)

Fig. 3 Consistent dysregulation of presynaptic and CASK-interacting genes in bulk RNA sequencing after 28 days of differentiation. a Upregulated and $\mathbf{b}$ downregulated gene sets emerging from GSEA in bulk RNA-sequencing data from mutation carriers compared with controls. c GSEA for CASK-interacting proteins (CASK PPI), ASD risk genes (SFARI), and NDD genes (NDD) in bulk RNA-sequencing data from mutation carriers compared with controls. d Network of CASK-interacting proteins with upregulation marked in blue and downregulation in red. e Venn diagram

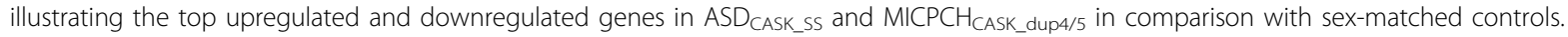
f Representative confocal microscopy images of neurons differentiated for 28 days and immunostained for Synapsin-1/2 (green), Homer-1 (red), and Hoechst (blue). Scale $=10 \mu \mathrm{m}$. $\mathbf{g}$ Quantification of Homer-1 and $\mathbf{h}$ Synapsin-1/2 particle size $(n=4$ per cell line). Statistical differences between cell lines were calculated using ANOVA with post hoc Tukey HSD. ${ }^{*} p<0.05,{ }^{* *} p<0.01,{ }^{* *} p<0.001$. Asterisks are color-coded according to case cell lines.

As expected from the increase in CASK expression during differentiation, we observed higher expression in differentiating neurons compared to NPCs (adj.p $=6 \mathrm{E}-6$, DESeq2, Supplementary Fig. 3d). Expression of the mutant RNA isoform does not cluster within one cell type (Fig. 2a), indicating that CASK protein expression changes are not cell-type specific.

\section{Reduced CASK levels alter presynaptic development}

We performed bulk RNA sequencing of day 28 maturing neurons from all cell lines to identify CASK-dependent expression changes. The RNA-sequencing data showed a similar distribution of CASK expression for $\mathrm{ASD}_{\text {CASK_SS }}$ and $\mathrm{MICPCH}_{\mathrm{CASKdup} 4 / 5}$ as earlier detected by RT-qPCR (Supplementary Fig. 4a). We also quantified the isoform-specific reads from exon-exon spanning fragments (Supplementary Table 2). Similar to RT-qPCR results at NES stage, we detected a low abundance of $C A S K$-mutant isoforms $C A S K_{14+}$ and $C A S K_{\triangle 14}$ of $4 \%$ and $8 \%$ in ASD $_{\text {CASK_SS, respectively. In addition, we detected }}$ both the mutant and reference nucleotide in $C A S K_{14+}$ reads that span the mutation site (Supplementary Fig. $4 \mathrm{~b}$ ). The de novo variant in DIAPH1 was present in around $50 \%$ of DIAPH1 transcripts (Supplementary Fig. 4c). In the $\mathrm{MICPCH}_{\mathrm{CASK} \text { dup } 4 / 5}$ transcriptome, we detected $14 \%$ $C A S K_{\text {dup } 4 / 5}$-specific sequence reads aligning to the exon 5-exon 4 junction. MICPCH $\mathrm{CASK}_{\text {_dup } 4 / 5}$ cells also showed downregulation of the XCI- regulating transcripts XIST and TSIX, which could explain activation of biallelic CASK expression (Supplementary Fig. 4d, e). In line with reduced expression of XCI transcripts, we observed an overall increase in $\mathrm{X}$-chromosome gene expression in the $\mathrm{MICPCH}_{\mathrm{CASK}_{\text {dup } 4 / 5}}$ cell line that was not present in ASD $_{\text {CASK_ss }}$ (Supplementary Fig. 4f, g). Principal component analysis (PCA) separated the mutation carrier cell lines from controls in the first component (Supplementary Fig. 4h), indicating that expression variability between cell lines is mostly explained by the CASK-mutation status. The second component of the PCA separated samples by sex.

With support from the PCA results, we performed a pooled analysis of mutation carrier transcriptomes to detect consistent CASK-related expression changes.
We detected 4098 differentially (BHA) and a $0.5 \log 2$ fold change (LC) (Supplementary Table 3). GSEA revealed the upregulation of genes involved in morphogenesis and development of different tissues, as well as extracellular matrix components and signaling pathways WNT and BMP (Fig. 3a). The downregulated categories consisted of presynaptic components, including the synaptic vesicle genes (Fig. 3b). In addition, we were specifically interested in the enrichment of genes involved in protein-protein interactions (PPI) with CASK (Pathway Commons), genes linked to ASD (SFARI scores 1, 2, and syndromic), and a compiled list of NDD genes (Supplementary Table 4). We detected significant enrichment of CASK PPIs in downregulated genes $(p=0.003, \mathrm{FDR}=0.003$, GSEA, Fig. 3c, Supplementary Table 5) and no enrichment in upregulated genes $(p=0.17, \mathrm{FDR}=0.25$, GSEA, Fig. 3c, Supplementary Table 5). Core enrichment genes are within a densely connected CASK PPI network, including MINT1 (encoded by $A P B A 1$ ), which is one of the tripartite binding partners of CASK (Fig. 3d). We separately looked at the nuclear interaction partners TBR1, CINAP (TSPYL2), and BCL11A that were not included in the obtained CASK PPI dataset. We found downregulation of TSPYL2 (LC $=-0.37, \quad$ p.adj. $=2 \mathrm{E}-3, \quad$ DESeq2 $)$ and $B C L 11 A$ (LC $=-0.53$, p.adj. $=1 \mathrm{E}-4$, DESeq2), and the absence of TBR1 expression in our model. Moreover, we detected a nominally significant enrichment of NDD genes in upregulated genes $(p=0.002, \mathrm{FDR}=0.25$, GSEA, Fig. 3c, Supplementary Table 5). In addition, we performed GSEA enrichment for individual cases versus sex-matched controls (Fig. 3c, Supplementary Tables 5-7), and found that CASK PPI and NDD genes were more enriched in the pooled analysis, indicating that this approach allowed us to detect consistent expression changes, independent of genetic backgrounds. Enriched upregulation is detected from sex chromosome bands $\mathrm{Xp} 22$ and $\mathrm{Xq} 22$, as well as cytoband $1 \mathrm{q} 44$ in $\mathrm{MICPCH}_{\mathrm{CASK}_{\text {_dup4/5 }}}$ (FDR of 0.003 , 0.005 , and 0.002 , respectively), and downregulated genes originating from cytoband $\mathrm{Yq} 11$ were enriched in ASD $_{\text {CASK_SS }}(\mathrm{FDR}=0.021)$.

In a complementary approach to the case-vs.-control analysis, we overlapped the most significant DEGs of case vs. sex-matched control analyses (Fig. 3e). Overrepresentation 
analysis in GO-term biological processes confirmed extracellular structure organization $(p<0.0001, \mathrm{FDR}<0.0001$, WebGestaltR, Supplementary Table 8 ) and synaptic vesicle cycle genes $(p<0.0001$, FDR $=0.0002$, WebGestaltR, Supplementary Table 8 ) as the most significant upregulated and downregulated process, respectively. The results from pathway analysis demonstrated that $C A S K$-mutant maturing neurons are deficient in expression of presynaptic CASKinteracting proteins.

We performed siRNA-mediated knockdown of CASK to verify $C A S K$-dependent expression changes during differentiation. We transfected male control cells after 1 day of differentiation with a pool of CASK-specific siRNAs or nontargeting control siRNA. Knockdown was efficient with a significant reduction of CASK mRNA and protein at day 28 (Supplementary Fig. 4i, j). GSEA of DEGs confirmed changes in synaptic vesicle pathway and extracellular matrix compounds (Supplementary Fig. 4k). Similar to DEGs from CASK-mutation carrier cell lines, NDD genes were nominally enriched in upregulated genes after CASK KD $(p=0.031, \mathrm{FDR}=0.58$, GSEA, Supplementary Table 9). In contrast to reduced expression of synaptic vesicle genes in the CASK-mutation carrier neurons, the genes underlying synaptic vesicle enrichment in response to CASK KD were upregulated. The conflicting direction of expression changes may be due to priming effects of reduced CASK in NES cells before differentiation.

To test if the dysregulation of presynaptic genes results in aberrant presynaptic development, we assessed synapse morphology in maturing neuronal cultures through immunofluorescence co-staining of pre- and postsynaptic markers Synapsin-1/2 and Homer-1, respectively (Fig. 3f). Synapsin-1/2 is part of the CASK PPI network (Fig. 3d), and the expression of SYN1 gene was downregulated (LC $=-1.08$, adj.p $=3.03 \mathrm{E}-13$, DESeq2). The Homer-1 gene, HOMER1 also showed downregulation ( $\mathrm{LC}=-0.33$, adj. $\mathrm{p}=0.002$, DESeq2). The number of Synapsin-1/2-positive presynaptic and Homer-1-positive postsynaptic regions did not reveal any difference between cell lines (Supplementary Fig. 5a). Similarly, the number of colocalizing regions, indicative of functional synapses, did not differ. While the Homer-1 particle size was comparable between cell lines (Fig. 3g), the mean Synapsin-1/2 particle size was

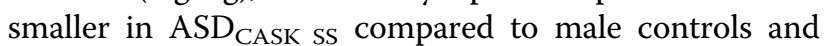
$\mathrm{MICPCH}_{\text {CASK_dup } 4 / 5}(p=0.021$ and $p=0.014$, respectively, ANOVA post hoc Tukey, Fig. 3h). Although dysregulation of presynaptic pathways was detected in the $\mathrm{MICPCH}_{\mathrm{CASK}_{-} \text {dup } 4 / 5}$ cell line, the presynaptic Synapsin-1/ 2 size was comparable to controls. The size differences indicated that reduced SYN1 expression affected pre-

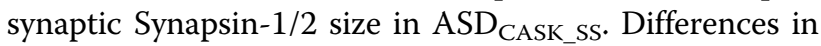
Synapsin-1 particle size and a more severe cellular phenotype in $\mathrm{ASD}_{\text {CASK_Ss }}$ are in line with the results obtained for CASK puncta.

\section{$\mathrm{E} / \mathrm{l}$ balance is affected by reduced CASK levels}

We explored the effect of the CASK mutations on the identified cell types using the BSEQ-sc analysis pipeline for gene expression deconvolution ${ }^{40}$. We used the cell-type markers generated from the single cell RNA sequencing data from $\mathrm{MICPCH}_{\mathrm{CASK} \text { dup } 4 / 5}$ cells to predict cell-type proportions underlying the bulk RNA transcriptomes. In our analysis, the estimated frequency of inhibitory GABA and undefined neuronal subtypes was unaffected in mutation carrier cell lines (Fig. 4a). Excitatory neurons were predicted with a lower frequency in both case cell lines, and NPCs were predicted with a higher frequency in

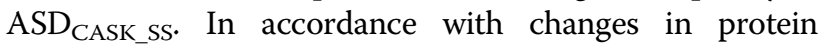
expression and morphological features, $\mathrm{ASD}_{\mathrm{CASK}_{\text {SS }}}$ showed a stronger effect than $\mathrm{MICPCH}_{\mathrm{CASK} \_ \text {dup } 4 / 5}$. The deconvolution of the CASK KD transcriptome predicted no change in cell-type proportions (Supplementary Fig. 5b).

Differences in cell-type proportions should be detectable on a morphological level. We performed immunofluorescence co-staining of the excitatory postsynapse marker Homer-1 with the presynaptic markers VGlut and VGAT as a proxy for excitatory and inhibitory presynapses, respectively. We had detected downregulation of SLC17A6 encoding for VGlut $(\mathrm{LC}=-1.38$, adj.p $=$ 2.44E-8, DESeq2) and $S L C 32 A 1$ encoding for VGAT (LC $=-2.44$, adj.p $=1.09 \mathrm{E}-37$, DESeq2) in the bulk RNA sequencing comparisons. VGlut particle number and size were comparable between cell lines (Fig. 4b, Supplementary Fig. 5c). The consistent number of VGlut particles per nuclei indicated comparable numbers of excitatory synapses. Staining of the inhibitory marker VGAT showed significant decrease in particle size between ASD $_{\text {CASK_ss }}$ and controls (ASD CASK_Ss $_{\text {vs. female }}$ control $p=0.005$ vs. male control $p=0.009$, ANOVA post hoc Tukey, Supplementary Fig. 6a, b). Co-staining of the inhibitory postsynaptic marker Gephyrin with VGAT in ASD $_{\text {CASK_SS }}$ confirmed reduced VGAT particle size

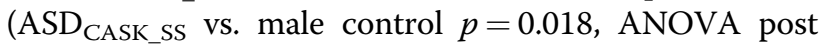
hoc Tukey, Fig. 4c, d). The size of colocalizing VGAT and Gephyrin particles, indicative of functional inhibitory synapses, was not significant (ASD CASK_Ss $_{\text {vs. male con- }}$ trol $p=0.067$, ANOVA post hoc Tukey, Supplementary Fig. 5d). In agreement with previous findings for CASK and Synapsin-1/2 particles, the number of presynaptic particles was unaffected in case cell lines (Supplementary Fig. 5c, d). Reduced VGAT particle size could indicate reduced inhibitory synapse size. Comparable VGAT presynaptic particles per nuclei indicate that inhibitory synapse numbers are comparable.

To compare our iPSC-derived neuronal data of reduced VGAT staining in inhibitory synapses to in vivo neurotransmitter concentrations, we investigated proton magnetic resonance spectroscopy $([1 \mathrm{H}] \mathrm{MRS})$ data, available for twin pairs including $\mathrm{ASD}_{\text {CASK_ss }}{ }^{43}$. We analyzed the 
A

\section{A Excitatory neurons}

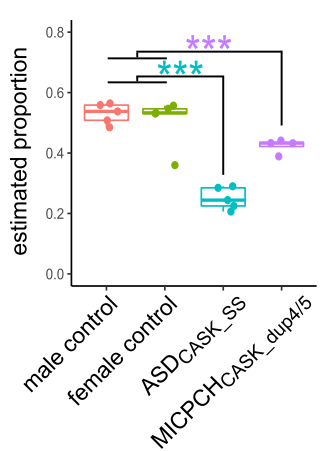

B

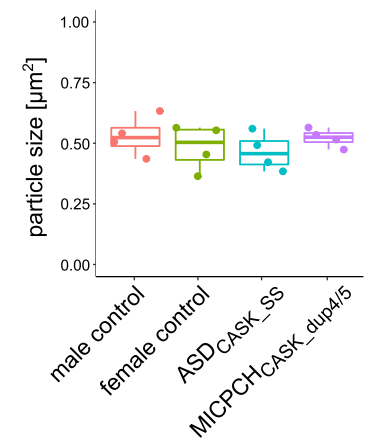

C

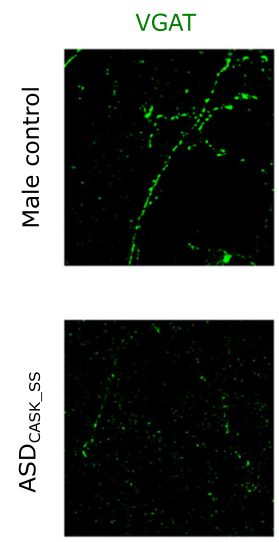

Inhibitory neurons

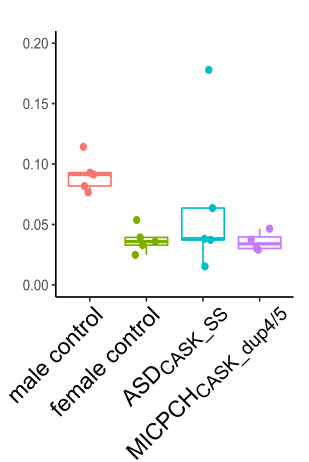

HOMER 1 size

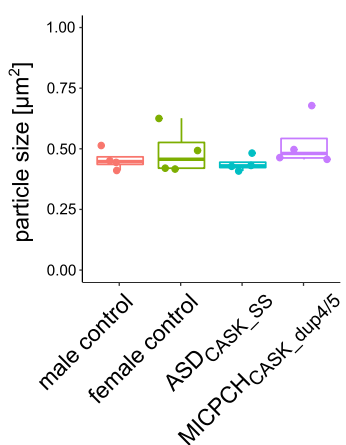

Neuroprogenitor

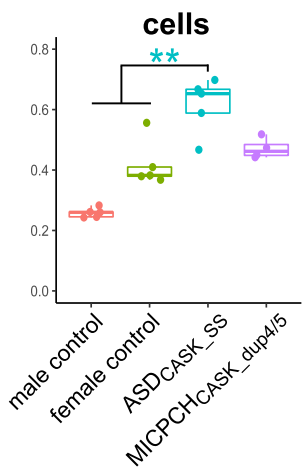

D Gephyrin size

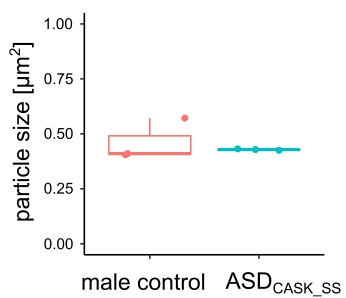

Undefined maturating neurons

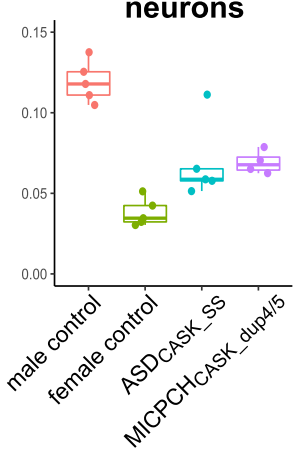

VGAT size

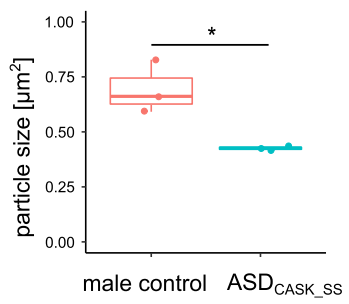

E GEPHYRIN VGAT/GEPH/HOECHST Zoom 3x
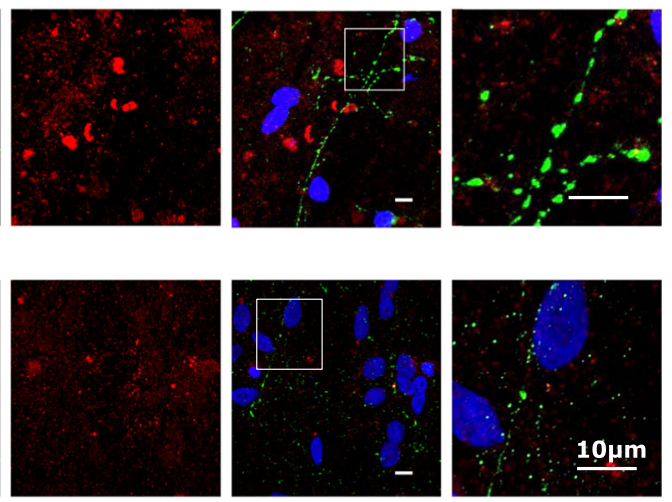

Fig. 4 Presynaptic effect is limited to inhibitory VGAT presynaptic marker. a Deconvolution of cell-type populations underlying bulk RNAsequence samples from all cell lines. b Quantification of VGlut and Homer-1 particle size ( $n=4$ per cell line). c Representative confocal microscopy images of neurons differentiated for 28 days and immunostained for inhibitory VGAT (green), Homer-1 (red), and Hoechst (blue). Scale $=10 \mu \mathrm{m}$. d Quantification of VGAT and Homer-1 particle size ( $n=3$ per cell line). Statistical differences between cell lines were calculated using ANOVA with post hoc Tukey HSD. ${ }^{*} p<0.05,{ }^{* *} p<0.01,{ }^{* * *} p<0.001$. Asterisks are color-coded according to case cell lines. e In vivo concentration of GABA in the DLPFC, MFC, and putamen of ASD CASK_SS $_{\text {S }}$ and his cotwin in relation to a typical developed control group.

GABA concentration in individuals without NDDs and obtained data for the dorsal medial prefrontal cortex (DLPFC, control $n=44$ ), medial frontal cortex (MFC, control $n=33$ ), and putamen (control $n=45$ ) (Supplementary Fig. 6a-d). The GABA concentration of the

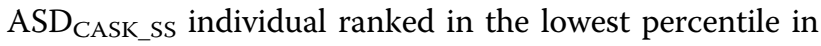
the DLPFC $(z=-1.16)$ and putamen $(z=-0.67)$ and in the 9th percentile of the MFC $(z=-0.86)$. GABA in the mutation carrier cotwin ranked similarly in the three brain regions (DLPFC: $z=-0.81$, putamen: $z=-0.67$, and MFC: $z=-0.97$, Fig. 4e).

Finally, we investigated spontaneous neuronal activity in the neuronal cultures. We imaged intracellular calcium release in 4- and 5-week-old neuronal cultures and compared calcium-signaling events between the cell lines. We identified spontaneously active neurons in 

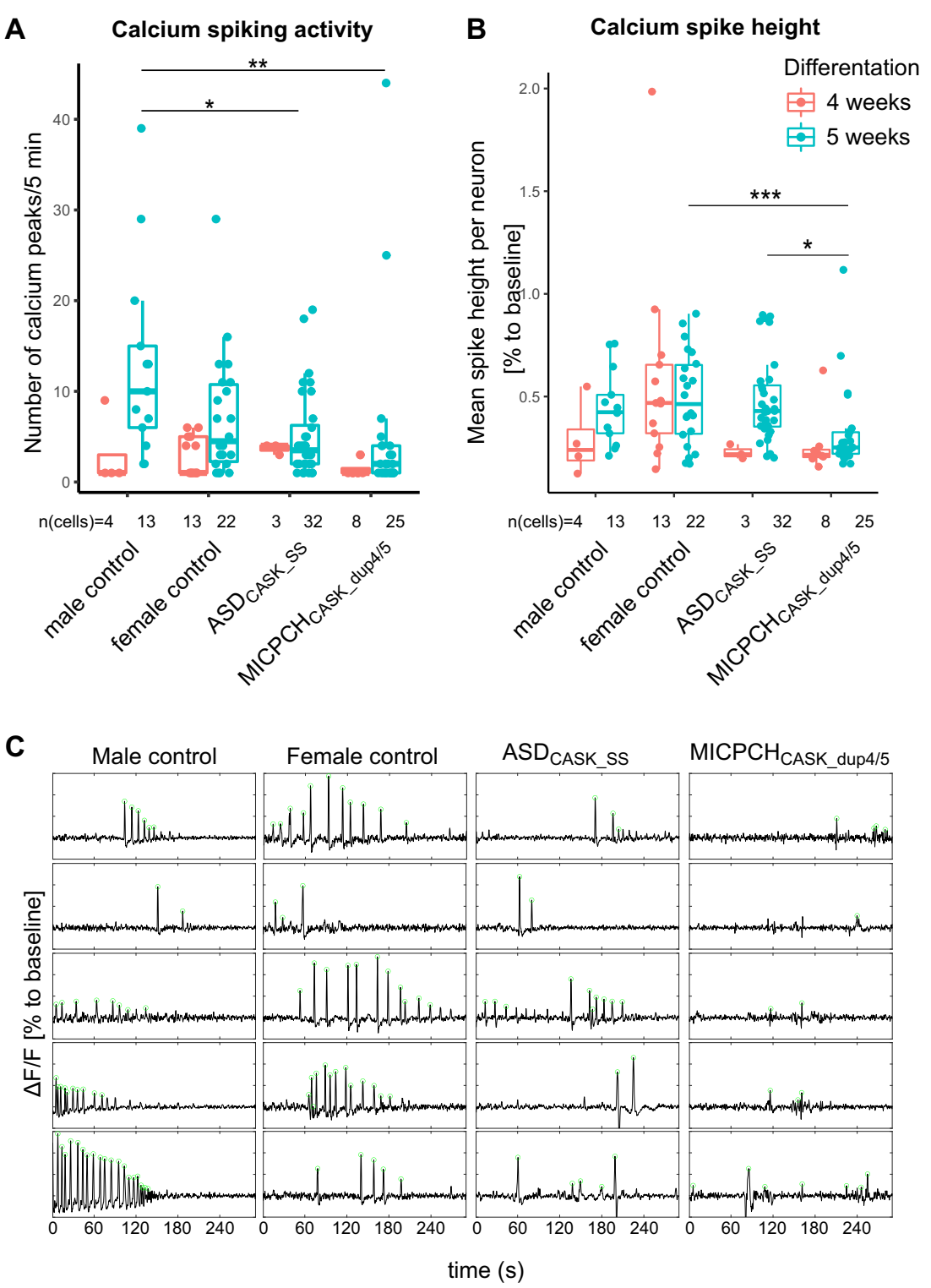

Fig. 5 Neuronal activity measured by time-lapse calcium imaging. a Number and $\mathbf{b}$ height of calcium spikes in active neurons of mutation carriers and controls differentiated for 4 and 5 weeks. Number of total neurons below the $x$-axis. Biological replicates at 4 weeks: female control $(n=2)$, male control $(n=2)$, ASD CASK_SS $_{2}(n=1)$, and MICPCH CASK_dup $4 / 5_{5}(n=2)$. Five weeks: female control $(n=2)$, male control $(n=2)$, ASD ${ }_{\text {CASK_SS }}$ $(n=5)$, and MICPCH CASK_dup4/5 $_{2}(n=2)$. Statistical differences between cell lines were calculated using pairwise Wilcoxon Rank Sum test followed by Bonferroni correction. ${ }^{*} p<0.05,{ }^{* *} p<0.01$. c Representative 5 -min traces of active neurons after 5 -week differentiation with called peak locations (green).

4-week-old cultures and observed an increased rate of calcium signals after 5 weeks (Fig. 5a, c, Supplementary Fig. $7 \mathrm{~g}$ ). No differences were detected between cell lines after 4 weeks of differentiation. At 5 weeks, ASD CASK_ss $_{\text {s }}$ and $\mathrm{MICPCH}_{\mathrm{CASK}_{\text {dup } 4 / 5}}$ showed less activity as compared with the male control (ASD CASK_Ss $p=0.0266$, $\mathrm{MICPCH} p=0.0019$, Bonferroni-corrected Pairwise Wilcoxon Rank Sum Test). In contrast to the mild cellular phenotype observed for $\mathrm{MICPCH}_{\mathrm{CASK} \_d u p 4 / 5}$, we observed aberrant calcium spike height (vs. female control $p=0.035$ vs. ASD CASK_ss $_{p}<0.001$, Bonferronicorrected Pairwise Wilcoxon Rank Sum Test, Fig. 5b). The rise and decay times are unaffected in $\mathrm{ASD}_{\mathrm{CASK}}$ SS and $\mathrm{MICPCH}_{\mathrm{CASK} \_d u p 4 / 5}$ (Supplementary Fig. 7e, f). In agreement with transcriptomic and morphological results, neuronal network activity was affected in ASD $_{\text {CASK_Ss }}$ with decreased firing rate and unaltered spike characteristics. 


\section{Discussion}

CASK-related disorders have emerged as an important genetic diagnosis for children with NDDs ranging from severely affected $\mathrm{MICPCH}$ patients to individuals with $\mathrm{ASD}^{1,4,6,7}$. The role of CASK as an essential player in synapse formation was reported previously using model organisms ${ }^{13,16,17}$; however, there is limited information of how the different CASK genetic variants found in humans affect the neuronal development. Therefore, we studied the consequences of two different mutations affecting CASK from individuals diagnosed with $\mathrm{MICPCH}$ and ASD. We utilized an iPSC- derived model to investigate the mutation effects during early neuronal maturation with detailed molecular, cellular, and functional characterization. We show that even slight changes in CASK expression in humans lead to dysregulation of the formation of presynapses, especially in inhibitory neurons. We suggest that the revealed presynaptic phenotype could be underlying the social difficulties seen in individuals affected by $C A S K$-related disorders, and that there are other mechanisms leading to the more profound brain malformations seen in more severely affected patients.

Using transcriptomic profiling of maturing neurons from CASK-mutation carriers and controls, we demonstrated that reduced CASK levels affected the transcription of presynaptic genes with significant enrichment for CASK-interacting partners. Dysregulation of CASKinteracting proteins in synapse gene networks is in line with transcriptomic profiling in heterozygous Caskknockout mice ${ }^{51}$. Importantly, downregulation of, e.g., NRXN family of genes, STXBP1, and SYT1 in CASKmutation carriers, provides a phenotypic link to other NDD genes. For instance, NRXN1 is one of the most studied NDD genes in which homozygous mutations cause Pitt-Hopkins-Like syndrome 2 characterized by NDDs, such as ID and $\mathrm{ASD}^{52}$, and heterozygous deletions predispose to several NDDs, psychiatric disorders, as well as congenital malformations ${ }^{53,54}$. Mutations in STXBP1 were first described in patients with early infantile epileptic encephalopathy, and are a common cause of epilepsy and encephalopathy with $\mathrm{ID}^{55,56}$. SYT1 mutations in Baker-Gordon syndrome cause infantile hypotonia, ophthalamic abnormalities, hyperkinetic movement disorders, motor stereotypies, and $\mathrm{DD}^{57}$. Disruption of this protein network in the developing presynapse could be underlying the behavioral phenotypes underlying CASKrelated NDDs. In addition to the presynapse, CASK has been implicated in functions within the dendrites, postsynapse, and neuronal nucleus. Although we observed the strongest enrichment in the presynapse, functional interaction partners of CASK in the other neuronal compartments were also dysregulated. We observed upregulation of CNTNAP2 and SDC2, which form complexes with CASK in dendrites to regulate postsynaptic development ${ }^{50,58}$. CNTNAP2 is linked to Pitt-HopinsLike syndrome 1 and increased ASD risk ${ }^{52,59,60}$. In addition, we observed downregulation of nuclear interacting partners BCL11A and TSPYL2, which have been linked to ID and ASD, respectively ${ }^{22,23}$. Taken together, CASK mutations cause dysregulation of NDD-related networks with a strong effect on presynapse development and putative impact on additional aspects of neuronal functions. Interestingly, we also illustrate upregulation of many developmental pathways in our analyses, such as mesenchyme, face and head development, as well as WNT signaling. Future studies should investigate these pathways in more detail and characterize how they might be linked to $C A S K$-related phenotypes, including microcephaly, scoliosis, and abnormalities of the eye and skin development ${ }^{1}$.

One benefit of the neuronal differentiation scheme used here was that we could investigate the effects on both inhibitory and excitatory neurons, and demonstrate that the transcriptional changes translate to reduced presynaptic size of inhibitory neurons as measured by Synapsin-1/2 and the inhibitory marker VGAT. The reduced presynaptic size, as measured by the selected markers and CASK puncta, may indicate aberrant synaptogenesis and synaptic function. In turn, this could lead to E/I imbalance. Earlier studies using electrophysiological measures in transgenic mice demonstrated decreased frequencies of mIPSCs and increased mEPSCs in adult CASK-deficient neurons ${ }^{16,17}$, establishing aberrant E/I balance as a phenotype underlying CASK deficiency. In addition, Mori et al. (2019) detected a decreased expression of the glutamate receptor GluN2B (encoded by Grin2b) in CASK-deficient neurons and concluded that postsynaptic CASK deficiency has an influence on E/I balance. In line with the previous findings, we detected reduced GRIN2B expression in $\mathrm{ASD}_{\text {CASK_Ss }}$ during neuronal maturation. However, we detected an unaffected number and size of excitatory VGlut synapses in contrast to the reduction in inhibitory presynaptic size, which together suggest that reduced inhibitory signaling is the primary mechanism underlying E/I imbalance in CASKdeficient neurons. Moreover, the low GABA concentration in all assessed brain regions of ASD CASK_ss $_{\text {and }}$ an cotwin could indicate that the inhibitory synapse effect is persistent during postnatal development. On the in vitro level, we observed reduced neuronal activity in the mutation carrier neurons. Changes in neuronal proportions, as suggested by the proportion estimates, could explain changes in neuronal network activity. However, consistent numbers of VGlut and VGAT punctae suggested stable cell-type proportions across cell lines. The observed decrease in VGAT size at GABAergic synapses could explain reduced bursting frequency. While a reduced inhibitory frequency would be expected to 
increase network activity, reduced bursting complexity could explain the reduced number of neuronal calcium events. In addition, GABA neurotransmitter function changes from excitatory to inhibitory during neural development and may have had an excitatory function in our model system ${ }^{61}$. Electrophysiological characterization of inhibitory and excitatory postsynaptic currents (IPSC and EPSC) in $\mathrm{ASD}_{\mathrm{CASK}_{\text {SS }}}$ and $\mathrm{MICPCH}_{\mathrm{CASK}_{\text {_dup }} / 5}$ neurons during neuronal maturation is necessary to link the observed changes in presynaptic marker size to changes in synaptic strength. E/I imbalance caused by aberrant neuronal firing was described for a variety of genetic mutations underlying NDD phenotypes. Studies investigating NRXN1 showed that similar to Cask KO mice, Nrxn1 KO mouse neurons remain with normal postsynaptic current amplitudes, but in contrast to Cask $\mathrm{KO}$ showed decreased mEPSC frequencies ${ }^{62}$. In human embryonic stem cell-derived neurons with heterozygous NRXN1 LoF mutation, increased CASK protein levels were observed together with decreased EPSC frequencies ${ }^{18}$ and homozygous deletions impair neuron maturation ${ }^{63}$, indicating that CASK-related pathways affect $\mathrm{E} / \mathrm{I}$ balance. In a recent study of isogenic human excitatory neurons disrupted for ASD-relevant genes, electrophysiological assessment demonstrated reduced spontaneous EPSCs and neuronal bursting in the presence of homo- or hemizygous mutations of $A T R X, A F F 2$, $K C N C 2, S C N 2 A$, and $A S T N 2^{64}$. Another study detected reduced neuronal firing in iPSC-derived neurons of eight individuals with idiopathic $\mathrm{ASD}^{65}$ and detected a correlation of reduced network complexity to behavioral and cognitive phenotype of the donors ${ }^{66}$. Moreover, reduced GABA concentration, as seen in the ASD CASK ss $_{\text {brain, }}$ was reported in groups of children with $\mathrm{ASD}^{67}$. Taken together, these findings show that CASK-related NDDs share the $E / I$ imbalance as pathological mechanisms with other ASD- and NDD-risk genes.

As an in vitro stem cell-based study, our results provide limited information in respect to the precise neurodevelopmental period and brain regions in which the observed effects contribute to the patient phenotype. We analyzed a short window of neuronal maturation and observed aberrant inhibitory presynaptic development in differentiated neurons. Longitudinal studies would be required to determine if these effects are persistent or represent delayed maturation. Postsynaptic effects, as well as effects at the excitatory neurons, have been described in models of CASK deficiency and certainly contribute to the phenotype range and severity in cases of $C A S K$-related disorders. Our study is limited to the characterization of the effects of reduced CASK protein levels, and it remains elusive how cellular phenotypes of CASK missense mutations compare. Thus, our results cover a limited part of the underlying molecular etiology.
In addition to the effects seen overall for the neuronal cultures, our study shows intriguing findings from the two unique mutations affecting $C A S K$. The hemizygous splicesite mutation detected in an autistic individual and his mild symptomatic monozygotic cotwin reduced expression of CASK $_{\mathrm{WT}}$ and caused splicing of two mutant mRNA isoforms. We excluded gain-of-function effects from mutant mRNA isoforms, as we detected only wild-type protein with similar cellular localization as in matched control neurons. Interestingly, we observed the rescue of the $C A S K_{\mathrm{WT}}$ splicing by an unknown mechanism. RNA sequencing of $\mathrm{ASD}_{\text {CASK_SS }}$ neurons indicated that the mutated $\mathrm{T} / \mathrm{U}$ is modified to the reference $\mathrm{G}$ nucleotide, suggesting that RNA editing is involved in the rescue mechanism. While reports on U-to-G RNA editing are rare, these mechanisms are shown to be relevant for synaptic transcripts and may be involved in the rescue of $C A S K_{\mathrm{WT}}$ in the $\mathrm{ASD}_{\text {CASK_SS }}{ }^{68,69}$. As both monozygotic twins carry the splice-site mutation and exhibit differences in symptom severity, it is interesting to speculate that environmental stressors could influence the rescue mechanisms and thus lead to variable severity. We have earlier studied pre- and perinatal environmental exposures in the RATSS twin sample, including the ASD CASK_SS $_{\text {case }}$ and his cotwin, and showed that uptake of essential metals zinc and manganese, and the neurotoxic metal lead, was associated with variable manifestation of autistic traits and ASD diagnoses ${ }^{70}$. These environmental stressors could potentially affect the RNA processing efficiency and mediate gene-environment interactions, leading to differences in outcomes of the twin pair ${ }^{68,71,72}$.

The instability of XCI has been reported in iPSCs and has hindered the cellular investigation of dominant $\mathrm{X}$ linked genes in NDDs. In the $\mathrm{MICPCH}_{\text {CASK_dup } 4 / 5}$ cells of the female donor, we observed mutant and wild-type CASK mRNA expression that likely resulted from XCI escape of the wild-type allele. Reports of XCI in adult human tissues did not indicate $C A S K$ as likely XCI escape gene $^{73}$. Heterozygous Cask-knockout mice that recapitulate the MICPCH phenotype showed Cask $_{\mathrm{WT}}$ expression in $50 \%$ of neurons, suggesting that XCI is stable in CASKnegative neurons ${ }^{17,74}$. The stability of XCI during iPSC reprogramming varies between protocols, and activation of both $\mathrm{X}$ chromosomes with subsequent random silencing of one $\mathrm{X}$ has been observed ${ }^{75}$. The milder phenotype in females carrying CASK mutations with skewed XCI suggests that the proportion of CASK-deficient cells is crucial for phenotype severity ${ }^{5,8}$. The escape of $C A S K_{\mathrm{WT}}$ in the $\mathrm{MICPCH}_{\mathrm{CASK} \text { dup } 4 / 5}$ cells likely rescues the cellular phenotype in our in vitro model, and we were unable to capture all pathoetiological mechanisms in the case with $\mathrm{MICPCH}$. Indeed, we consistently observed less severe cellular phenotypes in the $\mathrm{MICPCH}_{\mathrm{CASK} \_d u p 4 / 5}$ cells than

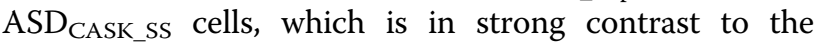


clinical phenotype severities of both disorders. This demonstrates the necessity to control for XCI pattern in iPSC studies of X-linked genes.

Currently, there are no drugs to treat $C A S K$-related disorders. A recent clinical study assessing neurorehabilitation effects on three girls with MICPCH and CASK mutations at the age of 2-4 years showed that the girls improved in motor skills, social interaction, and communication functions, indicating potential benefits of targeting the social aspects in $C A S K$-related disorders ${ }^{76}$. As we demonstrate a specific effect on the inhibitory presynapse and reduced GABA concentration in the cortical and subcortical brain regions of ASD $_{\text {CASK_SS, it is }}$ intriguing to speculate on the effect of GABA modulators in CASK-related disorders. Especially GABA agonists could be tested, which have shown positive effects on social deficits in ASD, and are in clinical trials for Fragile$\mathrm{X}$ syndrome ${ }^{77-79}$. Further development of drugs that target the presynapse and synaptic vesicle cycle could also be beneficial, but are currently not well explored ${ }^{80}$.

In conclusion, we show that reduced CASK protein levels affect presynaptic development and decrease inhibitory presynapse size, which may have consequences to $\mathrm{E} / \mathrm{I}$ balance in developing neural circuitries. Aberrant E/I balance, and synaptogenesis are two common biological pathways underlying NDDs of different genetic origin. Future pharmacological and clinical studies on targeting presynapses and E/I imbalance could lead to specific treatments for $C A S K$-related disorders.

\footnotetext{
Acknowledgements

The authors are grateful to the affected individuals and their families for their participation in this research. We also acknowledge the current and previous members of the RATSS team (Kerstin Andersson, Elodie Cauvet, Christina Coco, Johanna Ingvarsson, Elzbieta Kostrzewa, Johan Isaksson, Anna Råde, Annelies V'ant Westeinde, Elin Vahlgren, Karl Lundin, Martin Hammar, Lina Poltrago, Katell Mevel, and Steve Berggren, at the Center of Neurodevelopmental Disorders at Karolinska Institutet (KIND)). The authors acknowledge support from the National Genomics Infrastructure in Stockholm funded by Science for Life Laboratory, the Knut and Alice Wallenberg Foundation and the Swedish Research Council, the SNIC/Uppsala Multidisciplinary Center for Advanced Computational Science for assistance with massively parallel sequencing and access to the UPPMAX computational infrastructure, the Eukaryotic Single-Cell Genomics facility at the Science for Life Laboratory for assistance with SMARTSeq2 sequencing, and the iPS Core facility at Karolinska Institutet for assistance with the generation of IPSC and NES cells. The project was supported by the Swedish Research Council (E.H., A.F., S.B., and K.T.), Swedish Foundation for Strategic Research (A.F. and K.T.), The Swedish Brain Foundation-Hjärnfonden (E.H., A.F., S.B., and K.T.), the Harald and Greta Jeanssons Foundations (K.T.), Åke Wiberg Foundation (K.T.), Jerring Foundation (S.B.), the Swedish Order of Freemasons (S.B), Kempe-Carlgrenska Foundation (S.B), Sunnderdahls Handikappsfond (S.B.), Solstickan Foundation (C.W), the Pediatric Research Foundation at Astrid Lindgren Children's Hospital (S.B.), joint support from Swedish Research Council, Vinnova, Formas, and FORTE (S.B), the Innovative Medicines Initiative Joint Undertaking (grant agreement number 115300), which comprises financial contribution from the European Union's Seventh Framework Programme (FP7/2007-2013) and in-kind contributions from companies belonging to the European Federation of Pharmaceutical Industries and Associations (EU-AIMS), Strategic Research Area Neuroscience Stratneuro (K.T.), the L'Oréal-UNESCO for Women in Science prize in Sweden with support from the Young Academy of Sweden (K.T.), The Swedish Foundation for International Cooperation in Research and Higher Education STINT (K.T.) China
}

Scholarship Council (D.L.), Queen Silvia's Jubilee Fund (C.W. and D.L.), the Frederik and Ingrid Thurings Foundation (M.B.), and Barnavård Foundation (M.B., D.L., and K.T.) and Board of Research at Karolinska Institutet (K.T.). Open access funding provided by Karolinska Institute.

\section{Author details}

'Center of Neurodevelopmental Disorders (KIND), Division of Neuropsychiatry, Department of Women's and Children's Health, Karolinska Institutet, and Center for Psychiatry Research, Region Stockholm, Sweden. ${ }^{2}$ Astrid Lindgren Children's Hospital, Karolinska University Hospital, Region Stockholm, Sweden. ${ }^{3}$ Department of Women's and Children's Health, Karolinska Institutet, Stockholm, Sweden. ${ }^{4}$ Department of Psychiatry and Psychotherapy, Medical Center-University of Freiburg, Faculty of Medicine, University of Freiburg, Freiburg im Breisgau, Germany. ${ }^{5}$ Department of Radiology, Nationwide Children's Hospital, Columbus, OH, USA. Interdisciplinary Center for Neurosciences, Heidelberg University, Heidelberg, Germany. ${ }^{7}$ Gustavus Adolphus College, St. Peter, Minnesota, USA. ${ }^{8}$ Department of Neuroscience, Karolinska Institutet, Stockholm, Sweden. ${ }^{9}$ Department of Molecular Medicine and Surgery, Karolinska Institutet, Stockholm, Sweden. ${ }^{10}$ Department for Clinical Genetics, Karolinska University Hospital, Stockholm, Sweden. ${ }^{11}$ Department of Neuroradiology, Karolinska University Hospital and Department of Clinical Neuroscience, Karolinska Institutet, Stockholm, Sweden. ${ }^{12}$ Child and Adolescent Psychiatry, Stockholm Health Care Services, Stockholm County Council, Stockholm, Sweden. ${ }^{13}$ Curtin Autism Research Group, School of Occupational Therapy, Social Work and Speech Pathology, Curtin University, Perth, Western, Australia

\section{Author contributions}

M.B. and K.T. designed the study. J.W., C.W., L.M., E.H., S.B., and B.-M.A. performed acquisition of the phenotypic data, collection of biological samples, and provided administrative support. S.M., M-L.H., J.N., and R.S. generated and analyzed the MR and/or MRS data. M.K. and A.F. generated iPSCs and NES cells, and provided expertise in cell culture. M.B., F.M, I.R. V.M, L.B., and K.T performed genetic and molecular biology experiments. M.B. and F.M. performed immunostaining and confocal microscopy. M.B. and J.R. performed time-lapse calcium imaging with support from E.H., M.B., and F.M., and K.T. performed data and statistical analyses. E.H., A.F., S.B., and K.T. provided supervision in different parts of the study. All authors were part of interpreting the data and results. M.B., F.M., S.M., J.N., D.L., C.W., and K.T. prepared tables and figures. M.B. and K.T. drafted the paper. All authors performed a critical revision of the paper and approved the final version. M.B., S.B., A.F., and K.T. obtained funding.

\section{Conflict of interests}

The authors declare no competing interests. Sven Bölte declares no direct conflict of interest related to this article. Bölte discloses that he has in the last 5 years acted as an author, consultant, or lecturer for Shire/Takeda, Medice, Roche, Eli Lilly, Prima Psychiatry, and SB Education and Psychological Consulting $\mathrm{AB}$. He receives royalties for text books and diagnostic tools from Huber/Hogrefe, Kohlhammer, and UTB.

\section{Publisher's note}

Springer Nature remains neutral with regard to jurisdictional claims in published maps and institutional affiliations.

Supplementary Information accompanies this paper at (https://doi.org/ 10.1038/s41398-020-00994-0).

Received: 7 July 2020 Revised: 13 July 2020 Accepted: 22 July 2020 Published online: 14 September 2020

\section{References}

1. Deciphering Developmental Disorders S. Prevalence and architecture of de novo mutations in developmental disorders. Nature 542, 433-438 (2017).

2. Satterstrom, F. K. et al. Large-scale exome sequencing study implicates both developmental and functional changes in the neurobiology of autism. Cell 180, 568-584 (2020).

3. De Rubeis, S. et al. Synaptic, transcriptional and chromatin genes disrupted in autism. Nature 515, 209-U119 (2014). 
4. Moog, U. et al. Phenotypic and molecular insights into CASK-related disorders in males. Orphanet J. Rare Dis. 10, 44 (2015).

5. Moog, U. et al. Phenotypic spectrum associated with CASK loss-of-function mutations. J. Med. Genet. 48, 741-751 (2011).

6. Iossifov, I. et al. The contribution of de novo coding mutations to autism spectrum disorder. Nature 515, 216-U136 (2014).

7. Hayashi, S. et al. Comprehensive investigation of CASK mutations and other genetic etiologies in 41 patients with intellectual disability and microcephaly with pontine and cerebellar hypoplasia (MICPCH). PLOS ONE 12, e0181791 (2017).

8. Seto, T. et al. A novel CASK mutation identified in siblings exhibiting developmental disorders with/without microcephaly. Intractable Rare Dis. Res. 6 , 177-182 (2017).

9. LaConte, L. E. W. et al. Two microcephaly-associated novel missense mutations in CASK specifically disrupt the CASK-neurexin interaction. Hum. Genet. 137 231-246 (2018).

10. Gupta, A. R. et al. Rare deleterious mutations of the gene EFR3A in autism spectrum disorders. Mol. Autism 5, 31 (2014).

11. Stevenson, D., Laverty, H. G., Wenwieser, S., Douglas, M. \& Wilson, J. B. Mapping and expression analysis of the human CASK gene. Mamm. Genome 11, 934-937 (2000).

12. Hata, Y., Butz, S. \& Sudhof, T. C. CASK: a novel dlg/PSD95 homolog with an Nterminal calmodulin-dependent protein kinase domain identified by interaction with neurexins. J. Neurosci. 16, 2488-2494 (1996).

13. Butz, S., Okamoto, M. \& Sudhof, T. C. A tripartite protein complex with the potential to couple synaptic vesicle exocytosis to cell adhesion in brain. Cell 94, 773-782 (1998).

14. LaConte, L. E. et al. CASK stabilizes neurexin and links it to liprin-alpha in a neuronal activity-dependent manner. Cell Mol. Life Sci. 73, 3599-3621 (2016).

15. Lin, E. I., Jeyifous, O. \& Green, W. N. CASK regulates SAP97 conformation and its interactions with AMPA and NMDA receptors. J. Neurosci. 33, 12067-12076 (2013).

16. Atasoy, D. et al. Deletion of CASK in mice is lethal and impairs synaptic function. Proc. Natl Acad. Sci. USA 104, 2525-2530 (2007).

17. Mori, T. et al. Deficiency of calcium/calmodulin-dependent serine protein kinase disrupts the excitatory-inhibitory balance of synapses by downregulating GluN2B. Mol. Psychiatry 24, 1079-1092 (2019).

18. Pak, C. et al. Human neuropsychiatric disease modeling using conditional deletion reveals synaptic transmission defects caused by heterozygous mutations in NRXN1. Cell Stem Cell 17, 316-328 (2015).

19. Wang, G. S. et al. Transcriptional modification by a CASK-interacting nucleosome assembly protein. Neuron 42, 113-128 (2004).

20. Kuo, T. Y., Hong, C. J., Chien, H. L. \& Hsueh, Y. P. X-linked mental retardation gene CASK interacts with BCl11AVCTIP1 and regulates axon branching and outgrowth. J. Neurosci. Res. 88, 2364-2373 (2010).

21. Deriziotis, P. et al. De novo TBR1 mutations in sporadic autism disrupt protein functions. Nat. Commun. 5, 4954 (2014).

22. Moey, C. et al. Xp11.2 microduplications including IQSEC2, TSPYL2 and KDM5C genes in patients with neurodevelopmental disorders. Eur. J. Hum. Genet. 24, 373-380 (2016).

23. Dias, C. et al. BCL11A haploinsufficiency causes an intellectual disability syndrome and dysregulates transcription. Am. J. Hum. Genet. 99, 253-274 (2016).

24. Bölte, S. et al. The roots of autism and ADHD Twin Study in Sweden (RATSS). Twin Res. Hum. Genet. 17, 164-176 (2014).

25. Myers, L. et al. Minor physical anomalies in neurodevelopmental disorders: a twin study. Child Adolesc. Psychiatry Ment. Health 11, 57 (2017).

26. Wincent, J. et al. Copy number variations in children with brain malformations and refractory epilepsy. Am. J. Med. Genet. Part A 167, 512-523 (2015).

27. Stamouli, S. et al. Copy number variation analysis of 100 twin pairs enriched for neurodevelopmental disorders. Twin Res. Hum. Genet. 21, 1-11 (2018).

28. Richards, S. et al. Standards and guidelines for the interpretation of sequence variants: a joint consensus recommendation of the American College of Medical Genetics and Genomics and the Association for Molecular Pathology. Genet. Med. 17, 405-424 (2015).

29. Uhlin, E. et al. Derivation of human iPS cell lines from monozygotic twins in defined and xeno free conditions. Stem Cell Res. 18, 22-25 (2017).

30. Chambers, S. M. et al. Highly efficient neural conversion of human ES and iPS cells by dual inhibition of SMAD signaling. Nat. Biotechnol. 27, 275-280 (2009).

31. Falk, A. et al. Capture of neuroepithelial-like stem cells from pluripotent stem cells provides a versatile system for in vitro production of human neurons. PLOS ONE 7, e29597 (2012).
32. Dzyubenko, E., Rozenberg, A., Hermann, D. M. \& Faissner, A. Colocalization of synapse marker proteins evaluated by STED-microscopy reveals patterns of neuronal synapse distribution in vitro. J. Neurosci. Methods 273, 149-159 (2016).

33. Picelli, S. et al. Smart-seq2 for sensitive full-length transcriptome profiling in single cells. Nat. Methods 10, 1096-1098 (2013).

34. Li, W. V. \& Li, J. J. An accurate and robust imputation method sclmpute for single-cell RNA-seq data. Nat. Commun. 9, 997 (2018).

35. Butler, A., Hoffman, P., Smibert, P., Papalexi, E. \& Satija, R. Integrating single-cell transcriptomic data across different conditions, technologies, and species. Nat Biotechnol. 36, 411-420 (2018).

36. Reimand, J. et al. Pathway enrichment analysis and visualization of omics data using g:Profiler, GSEA, Cytoscape and EnrichmentMap. Nat. Protoc. 14, 482-517 (2019).

37. Subramanian, A. et al. Gene set enrichment analysis: a knowledge-based approach for interpreting genome-wide expression profiles. Proc. Natl Acad. Sci. USA 102, 15545-15550 (2005).

38. Shannon, P. et al. Cytoscape: a software environment for integrated models of biomolecular interaction networks. Genome Res. 13, 2498-2504 (2003).

39. Merico, D., Isserlin, R., Stueker, O., Emili, A. \& Bader, G. D. Enrichment map: a network-based method for gene-set enrichment visualization and interpretation. PLOS ONE 5, 11 (2010).

40. Baron, M. et al. A single-cell transcriptomic map of the human and mouse pancreas reveals inter- and intra-cell population structure. Cell Syst. 3, 346-360 e344 (2016)

41. Forsberg, D. et al. Functional stem cell integration into neural networks assessed by organotypic slice cultures. Curr. Protoc. Stem Cell Biol. 42, 2D 13 11-12D 1330 (2017).

42. Artimovich, E., Jackson, R. K., Kilander, M. B. C., Lin, Y. C. \& Nestor, M. W. PeakCaller: an automated graphical interface for the quantification of intracellular calcium obtained by high-content screening. BMC Neurosci. 18, 72 (2017).

43. Isaksson, J. et al. EU-AIMS Longitudinal European Autism Project (LEAP): the autism twin cohort. Mol. Autism 9, 26 (2018).

44. Edden, R. A. E., Puts, N. A. J., Harris, A. D., Barker, P. B. \& Evans, C. J. Gannet: a batch-processing tool for the quantitative analysis of gamma-aminobutyric acidedited MR spectroscopy spectra. J. Magn. Reson. Imaging 40, 1445-1452 (2014).

45. Harris, A. D., Puts, N. A. J. \& Edden, R. A. E. Tissue correction for GABA-edited MRS: considerations of voxel composition, tissue segmentation, and tissue relaxations. J. Magn. Reson Imaging 42, 1431-1440 (2015).

46. Carithers, L. J. et al. A novel approach to high-quality postmortem tissue procurement: the GTEx Project. Biopreserv. Biobank 13, 311-319 (2015).

47. Karczewski, K. J. et al. The mutational constraint spectrum quantified from variation in 141,456 humans. Nature 581, 434-443 (2020).

48. Neuhaus, C. et al. Extension of the clinical and molecular phenotype of DIAPH1-associated autosomal dominant hearing loss (DFNA1). Clin. Genet. 91, 892-901 (2017)

49. Al-Maawali, A. et al. Novel loss-of-function variants in DIAPH1 associated with syndromic microcephaly, blindness, and early onset seizures. Am. J. Med. Genet. Part A 170A, 435-440 (2016).

50. Gao, R. et al. CNTNAP2 stabilizes interneuron dendritic arbors through CASK. Mol. Psychiatry 23, 1832-1850 (2018).

51. Patel, P. A. et al. Haploinsufficiency of X-linked intellectual disability gene CASK induces post-transcriptional changes in synaptic and cellular metabolic pathways. Exp. Neurol. 329, 113319 (2020).

52. Zweier, C. et al. CNTNAP2 and NRXN1 are mutated in autosomal-recessive PittHopkins-like mental retardation and determine the level of a common synaptic protein in Drosophila. Am. J. Hum. Genet. 85, 655-666 (2009).

53. Dabell, M. P. et al. Investigation of NRXN1 deletions: clinical and molecular characterization. Am. J. Med. Genet. Part A 161a, 717-731 (2013).

54. Al Shehhi, M. et al. NRXN1 deletion syndrome; phenotypic and penetrance data from 34 families. Eur. J. Med. Genet. 62, 204-209 (2019).

55. Saitsu, H. et al. De novo mutations in the gene encoding STXBP1 (MUNC18-1) cause early infantile epileptic encephalopathy. Nat. Genet. 40, 782-788 (2008).

56. Uddin, M. et al. Germline and somatic mutations in STXBP1 with diverse neurodevelopmental phenotypes. Neurol. Genet. 3, e199 (2017).

57. Baker, K. et al. SYT1-associated neurodevelopmental disorder: a case series. Brain 141, 2576-2591 (2018).

58. Hu, H. T., Umemori, H. \& Hsueh, Y. P. Postsynaptic SDC2 induces transsynaptic signaling via FGF22 for bidirectional synaptic formation. Sci. Rep. 6, 33592 (2016). 
59. Bakkaloglu, B. et al. Molecular cytogenetic analysis and resequencing of contactin associated protein-like 2 in autism spectrum disorders. Am. J. Hum. Genet. 82, 165-173 (2008).

60. O'Roak, B. J. et al. Exome sequencing in sporadic autism spectrum disorders identifies severe de novo mutations. Nat. Genet. 43, 585-589 (2011).

61. Ben-Ari, Y. et al. Excitatory actions of gaba during development: the nature of the nurture. Nat. Rev. Neurosci. 3, 728-739 (2002).

62. Etherton, M. R., Blaiss, C. A., Powell, C. M. \& Sudhof, T. C. Mouse neurexin-1alpha deletion causes correlated electrophysiological and behavioral changes consistent with cognitive impairments. Proc. Natl Acad. Sci. USA 106, 17998-18003 (2009).

63. Lam, M. et al. Single cell analysis of autism patient with bi-allelic NRXN1-alpha deletion reveals skewed fate choice in neural progenitors and impaired neuronal functionality. Exp. Cell Res. 383, 111469 (2019).

64. Deneault, E. et al. Complete disruption of autism-susceptibility genes by gene editing predominantly reduces functional connectivity of isogenic human neurons. Stem Cell Rep. 11, 1211-1225 (2018).

65. Marchetto, M. C. et al. Altered proliferation and networks in neural cells derived from idiopathic autistic individuals. Mol. Psychiatry 22, 820-835 (2017)

66. Amatya, D. N. et al. Dynamical electrical complexity is reduced during neuronal differentiation in autism spectrum disorder. Stem Cell Rep. 13, 474-484 (2019).

67. Ford, T. C. \& Crewther, D. P. A comprehensive review of the (1)H-MRS metabolite spectrum in autism spectrum disorder. Front. Mol. Neurosci. 9, 14 (2016).

68. Dai, D. P. et al. Transcriptional mutagenesis mediated by 8-oxoG induces translational errors in mammalian cells. Proc. Natl Acad. Sci. USA 115 , 4218-4222 (2018).

69. Reid, J. G. et al. Mouse let-7 miRNA populations exhibit RNA editing that is constrained in the $5^{\prime}$-seed/ cleavage/anchor regions and stabilize predicted mmu-let-7a:mRNA duplexes. Genome Res. 18, 1571-1581 (2008).
70. Arora, M. et al. Fetal and postnatal metal dysregulation in autism. Nat. Com mun. 8, 15493 (2017).

71. Shomron, N., Malca, H., Vig, I. \& Ast, G. Reversible inhibition of the second step of splicing suggests a possible role of zinc in the second step of splicing. Nucleic Acids Res. 30, 4127-4137 (2002).

72. Dick, A. L. W. et al. Adenosine-to-inosine RNA editing within corticolimbic brain regions is regulated in response to chronic social defeat stress in mice. Front. Psychiatry 10, 277 (2019).

73. Tukiainen, $\mathrm{T}$. et al. Landscape of $\mathrm{X}$ chromosome inactivation across human tissues. Nature 550, 244-248 (2017).

74. Srivastava, S. et al. X-linked intellectual disability gene CASK regulates postnatal brain growth in a non-cell autonomous manner. Acta Neuropathol. Commun. 4, 30 (2016).

75. Dandulakis, M. G., Meganathan, K., Kroll, K. L., Bonni, A. \& Constantino, J. N. Complexities of $X$ chromosome inactivation status in female human induced pluripotent stem cells-a brief review and scientific update for autism research. J. Neurodev. Disord. 8, 22 (2016).

76. DeLuca, S. C., Wallace, D. A., Trucks, M. R. \& Mukherjee, K. A clinical series using intensive neurorehabilitation to promote functional motor and cognitive skills in three girls with CASK mutation. BMC Res. Notes 10, 743 (2017).

77. Erickson, C. A. et al. STX209 (arbaclofen) for autism spectrum disorders: an 8week open-label study. J. Autism Dev. Disord. 44, 958-964 (2014).

78. Erickson, C. A. et al. Brief report: pilot single-blind placebo lead-in study of acamprosate in youth with autistic disorder. J. Autism Dev. Disord. 44, 981-987 (2014).

79. Erickson, C. A. et al. Fragile $X$ targeted pharmacotherapy: lessons learned and future directions. J. Neurodev. Disord. 9, 7 (2017).

80. Li, Y. C. \& Kavalali, E. T. Synaptic vesicle-recycling machinery components as potential therapeutic targets. Pharm. Rev. 69, 141-160 (2017). 